\title{
A Routing and Scheduling Approach to Rail Transportation of Hazardous Materials with Demand Due Dates
}

\author{
Kan Fang ${ }^{\mathrm{a}}$, Ginger Y. Ke ${ }^{\mathrm{b}, *}$, Manish Verma ${ }^{\mathrm{c}}$ \\ ${ }^{a}$ College of Management and Economics, Tianjin University, Tianjin 300072, China. \\ ${ }^{b}$ Faculty of Business Administration, Memorial University of Newfoundland, St. John's, Newfoundland and \\ Labrador A1B 3X9, Canada. \\ ${ }^{c}$ DeGroote School of Business, McMaster University, Hamilton, Ontario L8S 4L8, Canada.
}

\begin{abstract}
This paper investigates the routing and scheduling of rail shipments of hazardous materials (hazmat) in the presence of due dates. In particular, we consider the problem of minimizing the weighted sum of earliness and tardiness for each demand plus the holding cost at each yard, while forcing a risk threshold on each service leg at any time instant. The Federal Railroad Administration (FRA) accident records, between 1999 and 2013, were analyzed to establish that train speed was the most significant factor in derailment. A mixed-integer programming model and a heuristic-based solution method are proposed for preparing the shipment plan. Finally, the analytical framework is used to study and analyze a number of realistic-sized problem instances generated using the infrastructure of a Class I railroad operator.
\end{abstract}

Keywords: Transportation; Railway; Hazardous Materials; Due Date; Train Speed

\section{Introduction}

A significant majority of hazardous materials (hazmat) shipments, including those essential to sustain our industrial lifestyle, are moved via the railroad network. For example, based on the latest commodity flow survey, railroad carried around 130 million tons of hazmat annually in the United States (DOT, 2007), whereas the number for Canada was 29 million tons (Provencher, 2008). The quantity of hazmat traffic on railroad networks is expected to increase significantly over the next decade, given the phenomenal growth of intermodal transportation and the growing use of rail-truck combination to move chemicals, and the more recent need to move an increasingly large number of crude oil shipments

${ }^{*}$ Corresponding author. Tel.: +1 709864 3469. E-mail address: gke@mun.ca (G.Y. Ke). 
from tight and shale formation regions in the United States and in Canada to the refineries located along the southern and eastern coasts of the continent (AAR, 2013; CAPP, 2014). It is true that railroads have a favorable safety statistic (Oggero et al., 2006), but the possibility of spectacular events resulting from multicar incidents, however small, does exist. For example, around 125 train accidents involving release from multiple tank cars have been reported over the last decade, which translates into an average of seven accidents every year (FRA, 2013). Most recently, the tragic event in Lac-Mégantic (Québec), in July 2013, is a reminder of the possible catastrophe associated with railroad transportation of hazmat.

It is important that though hazmat logistics experienced tremendous academic engagements over the past three decades, majority of the efforts were in the highway domain and thus not directly adaptable for rail shipments (Erkut et al., 2007). In this work, we provide a state-of-the art review of the relevant literature in Section 2, and propose the very first analytical framework that not only considers population exposure as the measure of hazmat risk, but also takes into consideration the equitable distribution of hazmat risk and the delivery due dates associated with rail hazmat demand. Thus, the proposed work has the following contributions. First, an empirical analysis is conducted on rail accidents dataset to reveal the cause-and-effect relationship between train speed and derailment risk. Second, we propose an optimization model for routing and scheduling rail hazmat shipments such that customer demands are satisfied within specified time windows while adhering to the risk thresholds (i.e., spatial distribution of risk) on different service legs. Third, we prove the complexity of the resulting mathematical program, and then develop a heuristic-based algorithm to solve it. Finally, the proposed framework was applied to several problem instances generated using realistic railroad networks, and the resulting analyses revealed non-negligible cost savings either by improving the quality of rail tracks or by imposing a higher risk thresholds on transportation corridors.

\section{Literature review}

Since the proposed investigation transcends multiple sub-areas within the hazmat transportation domain, we organize the literature review under four streams: risk assessment; risk management; distribution of risk or equity; and, delivery due dates.

Risk assessment: Risk is most commonly defined as the product of the probability and the consequence of an undesirable event (Covello and Merkhofer, 1993). This expected consequence approach has been used in a number of works to mostly evaluate risk from highway transport of hazmat and that from fixed facilities (Erkut and Verter, 1998; Alp, 
1995). The challenges associated with obtaining reliable data motivated the development of two alternative measures for hazmat risk. The first measure (i.e., incident probability) does not include consequence and thus is appropriate for hazmat with relatively small danger zones (Saccomanno and Chan, 1985; Abkowitz et al., 1992). On the other hand, the second measure (i.e., population exposure) focuses on just consequence, and more specifically on the total number of people exposed to the possibility of an undesirable consequence due to a hazmat shipment (Batta and Chiu, 1988a; ReVelle et al., 1991). It was suggested that the last measure constitutes a worst-case approach to transport risk, and hence would be particularly suitable for assessing risk as perceived by the public, as well as for estimating required emergency response capability. We use the last measure of risk in this paper. For expositional reasons, we have only listed the seminal papers and invite the interested readers to refer to Verma and Verter (2013) and Bagheri et al. (2014) for a state of the art review on railroad transportation of hazmat.

Risk management: and/or mitigation within the rail transportation domain would include techniques to improve rail-track safety, railcar tank designs, and routing models. Glickman and Rosenfield (1984) made use of the past derailment data to derive various forms of risk, i.e., total number of fatalities, and frequency of accidents. Barkan et al. (2003) conducted a statistical analysis of the railroad accident data to conclude that the speed of derailment and the number of derailed railcars are highly correlated with hazmat release, and then proposed estimates of direct and conditional probabilities in conducting risk analysis (Anderson and Barkan, 2004). The last 20 years has also witnessed a number of efforts geared towards risk mitigation with a focus on reducing the frequency of tank car accidents and the likelihood of releases. While Raj and Pritchard (2000) reported that DOT-105 tank car design is safer than the DOT-111 type, Barkan et al. (2000) showed that tank cars equipped with surge pressure reduction devices experienced lower release rates than those without. More recently, a metric to assess the performance of a tank car in an accident was developed (Saat and Barkan, 2005), followed by analyses of the tradeoff between increased damage resistance and greater exposure to accidents (Barkan and Ukkusuri, 2007), and that between weight and safety (Barkan, 2008).

Though train routing and scheduling issues are crucial to freight train operations (Cordeau et al., 1998), hazmat related engagements are rather few. Glickman (1983) was the first to point out the importance of considering population exposure risk when dealing with hazmat shipments. The issue of (re)routing was revisited following the terrorist attacks of 9/11. Saat and Barkan (2006) evaluated transport risk for different routes by taking into 
consideration the issue of re-routing and appropriate tank car design, while Glickman et al. (2007) combined transportation modeling with risk assessment to study a sample of intercity routes and to identify opportunities for improving safety at a reasonable cost. Finally, Verma (2009) and Verma et al. (2011) proposed analytical approaches that make use of both transport cost and transport risk to prepare routing plans for rail hazmat shipments. Note that while the impact of scheduling hazmat trucks on risk has been explored over the past two decades (see for example, Nozick et al., 1997; Miller-Hooks and Mahmassani, 1998), no similar effort has been in the railroad domain.

Distribution of risk: or equity has received increased attention from hazmat researchers over the past decade, including some recent efforts resulting in the development of sophisticated leader-follower strategies. To this end, Gopalan et al. (1990a,b) proposed models for highway hazmat shipments that would minimize total risk while guaranteeing equitable risk spreading; Linder-Dutton et al. (1991) focused on the global route planning problem involving multiple origin-destination pairs; Akgün et al. (2000) aimed to find dissimilar paths between an origin-destination pair; Bell (2006) emphasized on the minimization of the maximum link risk; Carotenuto et al. (2007a) concerned the minimization of total risk with bounded maximum link risk; Carotenuto et al. (2007b) proposed a tabu search algorithm to the routing and scheduling of hazmat shipments so as to provide an equitable distribution of the risk; and, Kang et al. (2014) developed a generalized hazmat routing planning model considering the risk equity under a Value-at-Risk framework. For a review of equitably locating hazmat facilities, see Romero et al. (2016), which employed the Gini coefficient to describe the risk equity in making facility location and routing decisions. Most recently, methodologies motivated by game-theory have been proposed to ensure a more equitable hazmat transport network (see for example, Verter and Kara, 2008; Bianco et al., 2009, 2016). It is important to note that all the efforts have been in the highway shipment context, and that we are not aware of any development for rail hazmat shipments.

Delivery due dates and delivery time-windows: sub-area has witnessed significant research engagements over the past three decades in all modes of transportation, but particularly in highway shipments of regular freight (see for example, Kallehauge, 2008). Kwon et al. (1998) is a notable example in the railroad domain, while Christiansen and Fagerholt (2002) is an example in the marine transportation domain. In order to ensure that all demands can be met in time and with minimum delivery cost, researchers have widely adopted the objective of minimizing the linear combination of earliness, tardiness, plus some other costs (e.g. the holding cost), both in scheduling and supply chain management 
(see for example, Bülbül et al., 2004; Wang et al., 2004; Agustina et al., 2014). While these engagements were in relation to regular freight, Verma and Verter (2010); Verma et al. (2012) and Assadipour et al. (2015) are the only peer-reviewed works on hazmat freight.

Thus, we make a first attempt to develop an analytical framework that makes use of population exposure as the measure of hazmat risk, and also considers the (equitable) distribution of risk and delivery due dates. Hereafter, we refer to this problem as rail transportation of hazardous materials with demand due dates (RTHD). The rest of the paper is organized as follows. Section 3 contains an analysis of the derailment data from the Federal Railroad Administration to identify the most important causal factor, followed by the development of the mixed-integer programming model in Section 4. Based on the lower bounding scheme developed in Section 5, a heuristic algorithm is proposed in Section 6 for finding feasible solutions to the RTHD problem. Section 7 tests the computational performance of the mathematical programming and the heuristic algorithm on instances arising from random generated demands and real-life rail networks. Finally, we conclude our research and provide possible future research directions in Section 8.

\section{Primary determinant of rail derailment}

This section is intended to independently investigate the impact of various factors on the number of railcars derailed, though we are familiar with the earlier efforts such as Anderson and Barkan (2004), Barkan et al. (2003), Liu et al. (2012), and Verma (2011). Our objective was two-fold: first, focus only on the mainline operations of the Class I railroad operators, who are responsible for moving majority of hazmat shipments; and second, analyze the more recent data (i.e., from 1999 to 2013) to identify any changes or the need for modifying the existing expressions for estimating the number of railcar derailed.

A total of 5476 derailment accident records, over the indicated fifteen year period, were parsed and prepared for conducting associative analyses. The FRA dataset suggests four possible visibility levels (i.e., $v \in\{$ dawn, day, dusk, dark $\}$ ), six weather levels (i.e., $w \in$ $\{$ clear, cloudy, rain, fog, sleet, snow $\}$ ), and seven track class levels (i.e., $c \in\{X, 1,2, \ldots, 6\}$ ). In addition, for each accident, information is provided on train speed, train length and number of cars derailed. Note that barring two instances, the train speed is less than 70 miles per hour. Hence, for expositional ease, we categorize train speeds into seven posssible intervals, i.e., $\{[0,10),[10 i, 10(i+1))\}$, where $i=\{1,2, \ldots, 6\}$. For example $(v, w, c, s)$ would indicate an accident scenario with visibility $v$, weather $w$, track class $c$, and train speed belonging to one of the above train speed intervals $s$. The 5474 accident records 
were aggregated into 371 subgroups following the above categorization. Finally, for each subgroup, the average train speed, average train length, and average cars derailed were determined. Note that both $v, w$ and $c$ have multiple categorical levels, and hence dummy variables were introduced to capture their impact in the resulting multiple regression model.

Table 1: Regression results for number of cars derailed

\begin{tabular}{|c|c|c|c|c|c|c|c|c|}
\hline \multirow{2}{*}{ Variables } & \multicolumn{4}{|c|}{ With Visibility (371 subgroups) } & \multicolumn{4}{|c|}{ Without Visibility (147 subgroups) } \\
\hline & Coefficient & Std. Error & $t$-value & & Coefficient & Std. Error & $t$-value & \\
\hline Intercept & -4.91194 & 3.58004 & -1.372 & & -11.97839 & 5.51585 & -2.172 & * \\
\hline Train speed & 0.23730 & 0.02964 & 8.007 & $* * *$ & 0.30299 & 0.04475 & 6.770 & $* * *$ \\
\hline Train length & 0.07559 & 0.02183 & 3.462 & $* * *$ & 0.15098 & 0.04200 & 3.594 & $* * *$ \\
\hline W1 (Cloudy) & 0.66382 & 1.27551 & 0.520 & & 0.75012 & 2.27377 & 0.330 & \\
\hline W2 (Rain) & 3.45853 & 1.38784 & 2.492 & * & 3.42965 & 2.36484 & 1.450 & \\
\hline W3 (Fog) & 0.97036 & 1.81230 & 0.535 & & 2.54615 & 2.56077 & 0.994 & \\
\hline W4 (Sleet) & -2.46518 & 2.87582 & -0.857 & & -4.03648 & 3.33948 & -1.209 & \\
\hline W5 (Snow) & 3.62156 & 1.47486 & 2.456 & $*$ & 5.13641 & 2.40631 & 2.135 & * \\
\hline T1 (Track 1) & 1.02782 & 3.25935 & 0.315 & & -1.23994 & 4.78698 & -0.259 & \\
\hline T2 (Track 2) & 4.54013 & 3.09288 & 1.468 & & 4.47482 & 4.48328 & 0.998 & \\
\hline T3 (Track 3) & 1.44693 & 3.06624 & 0.472 & & -0.61074 & 4.48104 & -0.136 & \\
\hline T4 (Track 4) & -0.98216 & 3.07180 & -0.320 & & -3.75385 & 4.46105 & -0.841 & \\
\hline T5 (Track 5) & -0.61795 & 3.12750 & -0.198 & & -4.52211 & 4.50356 & -1.004 & \\
\hline T6 (Track 6) & -4.84878 & 4.51913 & -1.073 & & -7.36563 & 5.64791 & -1.304 & \\
\hline V1 (Day) & -1.21818 & 1.37564 & -0.886 & & - & - & - & \\
\hline V2 (Dusk) & -1.30206 & 1.54229 & -0.844 & & - & - & - & \\
\hline V3 (Dark) & -0.29369 & 1.33966 & -0.219 & & - & - & - & \\
\hline$R^{2}$ & & 0.2135 & & & & 0.3513 & & \\
\hline
\end{tabular}

*: the level of statistical significance of the variables

It is clear from the first block in Table 1 (i.e., with visibility) that train speed, train length, and two weather levels (i.e., rain and snow) have significant impact on the number of cars derailed. It was interesting to note that visibility had no bearing on the number of railcars derailed; and this fact perhaps points to the technological advancements that have rendered this variable unimportant. This also encouraged us to re-aggregate the dataset without visibility considerations, which resulted in 147 subgroups (i.e., second block in Table 1). It is obvious that train speed is the determinant factor in estimating the number of railcars derailed, and hence we consider just that moving forward. Also note that both train speed and train length influence derailment, yet we focus only on train speed because the length of the train depends on the number of railcars, i.e., endogenous to the optimization model. Equation (1) depicts a simple linear relationship between the proportion of cars derailed (i.e. number of cars derailed/total number of cars) and train speed,

$$
P(D \mid A)=0.0028 s+0.043
$$


where $P(D \mid A)$ is the probability of derailment of a railcar $(D)$, given a train accident $(A)$ occurs.

\section{Hazmat shipments planning}

An instance of the RTHD problem consists of a rail network $\mathcal{G}=(\mathcal{C}, \mathcal{L})$, where $\mathcal{C}$ is the set of yards and $\mathcal{L}$ is the set of service legs (all tracks on which a train travels nonstop between two yards). Each yard $c \in \mathcal{C}$ has a required processing time $q_{c}$ for classification or transfer processes. There is a discrete set of speeds $\mathcal{S}=\left\{s_{1}, \ldots, s_{d}\right\}$ : a train running at speed $s \in \mathcal{S}$ on service leg $\ell$ has an associated risk per hazmat railcar $r_{\ell s}$ and traveling time $p_{\ell s}=\left\lceil p_{\ell} / s\right\rceil$, where $p_{\ell}$ is the distance between two consecutive yards that are connected by service leg $\ell$, and $\lceil\cdot\rceil$ is the ceiling function. Without loss of generality, we assume that $s_{1}<s_{2}<\cdots<s_{d}$.

There is a set of demands $\mathcal{M}$, in which each demand is characterized by its origin and destination yards. For each demand $m \in \mathcal{M}$, the set of its available itineraries $I^{m}$ is given, where we define an itinerary as a feasible journey of a demand from the origin yard to the destination yard, including the yard and service leg operations. In addition, each yard is visited exactly once in each itinerary.

Within a given time horizon, we let $\mathcal{T}=\{0,1,2, \ldots, T\}$ be the set of time instances within it. In addition, each demand $m \in \mathcal{M}$ has a due date $d_{m}$ : when the demand is met before (after) its due date, an earliness (tardiness) penalty cost $\theta_{m}\left(\psi_{m}\right)$ per unit time and per railcar is incurred. In addition, when the train arrived at yard $c \in \mathcal{C}$ but keep the railcars idle (i.e. idle without classification or transfer process), a holding penalty cost $\eta_{c}$ per unit time and per railcar is incurred. The number of hazmat and regular railcars required for each demand $m \in \mathcal{M}$ are $h_{m}$ and $\bar{h}_{m}$ respectively. Depending on the track quality of each service leg $\ell$, there is a maximum speed $s_{\ell}$ that a train can run. In addition, we are given a risk threshold $R_{\max }$ that the railroad carrier can reach on each service leg at any time instant.

In this paper, we focus on minimizing the total cost, i.e., the earliness, tardiness and holding costs incurred to fulfill all the demands. We define a feasible routing and scheduling plan in which each demand is met by one of its available itineraries and trains are scheduled appropriately so that the given risk threshold $R_{\max }$ can be satisfied on each service leg at any time instant.

We make the following assumptions in this paper. First, each demand will be met using exactly one of the available itineraries. More specifically since railroad industry operates on 
consolidation, this assumption would ensure that demand is not split during transportation. Second, a freight train runs at a constant speed on a given service leg at any time instant. That is, if the track class for a service leg does not change, a train entering this service leg would run at a constant speed until arriving at the destination yard that is at the end of the service leg. Third, higher train speed implies higher risk. Note that this assumption is justified by the statistical analysis on the FRA dataset in Section 3. Fourth, at least one feasible routing and scheduling plan for each demand exists, which implies that the risk threshold $R_{\max }$ cannot be too small such that no feasible plan exists. This assumption is motivated by the need to meet customer demand. Fifth, rail networks have siding tracks to enable faster and higher priority trains to overtake the slower or lower priority ones. Sixth, for the simplicity of analysis with little loss of applicability, we assume that different freight trains can run at different speeds on the same service leg even when they depart at the same time instant. The reason for applying such assumption is based on the following two observations. 1) Generally, trains can depart a yard on different siding tracks, and after traveling for a short distance the tracks merge to the same mainline. 2) When the trains are scheduled to depart according to a nondecreasing order of their speeds, then the distance between a faster and a slower trains will soon be greater than the radius of the danger zones, and thus they do not affect each other on the risk perspective. Seventh, we assume wind direction and speed that would result in the most conservative risk estimates for the given transportation corridors. This assumption enables us to provide the most conservative estimates, and the relevant operational details are provided in Section 7.1. Eighth, we assume that the total risk of trains that departs the same service leg simultaneously at the same speed is a linear combination of the risk induced by each hazmat railcar of these trains. Finally, we assume that yard risk resulting from low train speeds is not significant, and thus is ignored.

\subsection{A rail transportation system}

Before developing the mathematical model, and for expositional reasons, we next outline a simple example to illustrate the cost and risk issues in the RTHD problem. Figure 1 represents a rail network $\mathcal{G}=(\mathcal{C}, \mathcal{L})$, where $\mathcal{C}=\{A, B, C, D\}$ and $\mathcal{L}=\left\{\ell_{1}, \ell_{2}, \ldots, \ell_{6}\right\}$. Given the definition of an itinerary, the origin and destination pair $(A, D)$ shown in Figure 1 can be met by the following itineraries:

- itinerary $k_{1}: A \stackrel{\ell_{4}}{\longrightarrow} D$;

- itinerary $k_{2}: A \stackrel{\ell_{1}}{\longrightarrow} B \stackrel{\ell_{5}}{\longrightarrow} D$; and 
- itinerary $k_{3}: A \stackrel{\ell_{1}}{\longrightarrow} B \stackrel{\ell_{2}}{\longrightarrow} C \stackrel{\ell_{3}}{\longrightarrow} D$;

while the journey of $A \stackrel{\ell_{1}}{\longrightarrow} B \stackrel{\ell_{2}}{\longrightarrow} C \stackrel{\ell_{6}}{\longrightarrow} A \stackrel{\ell_{4}}{\longrightarrow} D$ is not a feasible itinerary since yard $A$ is visited twice.

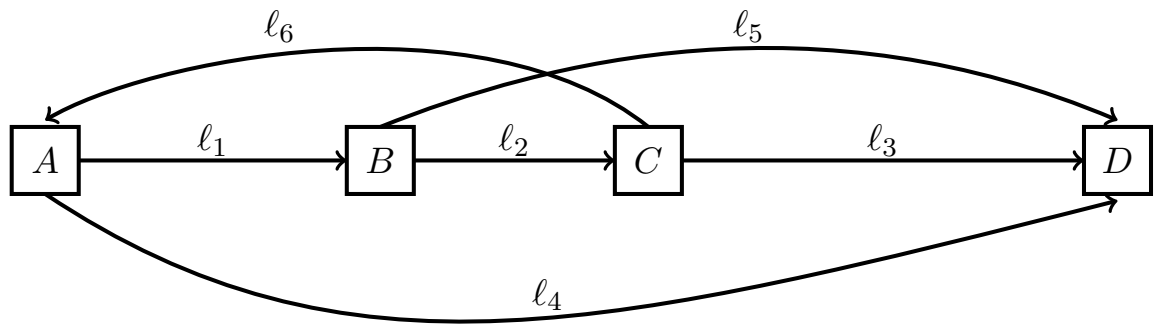

Figure 1: An example of a rail network.

We denote $n_{k}$ as the number of yards in itinerary $k$, and thus the corresponding number of service legs in itinerary $k$ is $n_{k}-1$. We define $\alpha_{\ell k}^{i}=1$ if service leg $\ell$ is the $i$ th leg in itinerary $k$, and 0 otherwise; $\gamma_{c k}^{i}=1$ if yard $c$ is the $i$ th yard in itinerary $k$, and 0 otherwise. For example, in itinerary $k_{1}$, service leg $\ell_{4}$ is the first leg and yard $D$ is the second yard, so we have $\alpha_{\ell_{4} k_{1}}^{1}=1$ and $\gamma_{D k_{1}}^{2}=1$. It is obvious that once a rail network is given, the values of $\alpha_{\ell k}^{i}$ and $\gamma_{c k}^{i}$ are fixed.

In order to fulfill a demand, the railroad carrier needs to choose one of its available itineraries to deliver the materials. In addition, the carrier also has to decide when to depart at each yard (i.e. determine if additional idle time should be imposed to the trains) and at which speed the train should run on a service leg after departing a yard in order to minimize the total cost, under the restriction on risk threshold on each service leg at any time instant.

As mentioned above, we impose a risk threshold $R_{\max }$ on each service leg at any time instant to ensure the equity in the distribution of risk. The reason for applying such criteria is based on three aspects. First, from our second assumption, once a train starts running on a service leg, we cannot change its speed until it arrived at the destination yard of this service leg. Therefore, the corresponding risk will keep the same along this service leg. Second, as a result of our fifth assumption, when a faster train passes a slower one, the railroad carrier will pull over and stop the slower one to a siding track section for a short time, and free the way for the faster one. Third, from our sixth assumption, when a faster 
and a slower trains depart at the same time instant on the same service leg, they do not affect each other. For the sake of simplicity with little loss of generality, in this work, we assume that the risk incurred by trains starting at different time instances or running at different speeds are independent, that is, we do not accumulate their risk together since these trains affect different segments of the service leg during their transportation. Based on these three assumptions, we only need to keep track of the risk incurred by a train at its starting time instant on each service leg at each speed. That is, let $R(\ell, s, t)$ be the risk incurred by trains departing on service leg $\ell$ at speed $s$ at time instant $t$, then we must have $R(\ell, s, t) \leq R_{\max }$ for $\ell \in \mathcal{L}, s \in \mathcal{S}, t \in \mathcal{T}$. To improve the readability of this paper, we list the notation involved above in Table 2 .

Table 2: Notation summarization

\begin{tabular}{ll}
\hline Sets & \\
\hline $\mathcal{C}$ & Set of yards, indexed by $c$. \\
$\mathcal{L}$ & Set of service legs, indexed by $\ell$ \\
$\mathcal{S}$ & Set of speeds, indexed by $s$. \\
$\mathcal{M}$ & Set of demands, indexed by $m$. \\
$I^{m}$ & Set of available itineraries for demand $m$, indexed by $k$. \\
$\mathcal{T}$ & Set of time instances within the given time horizon, indexed by $t$. \\
\hline Parameters & \\
\hline$q_{c}$ & Processing time for classification or transfer process at yard $c$. \\
$r_{\ell s}$ & Risk per hazmat railcar for a train runs at speed $s$ on service leg $\ell$. \\
$p_{\ell}$ & Distance between two consecutive yards that are connected by service leg $\ell$. \\
$p_{\ell s}$ & Traveling time for a train runs at speed $s$ on service leg $\ell$. \\
$d_{m}$ & Due date for demand $m$. \\
$\theta_{m}$ & Earliness penalty cost per unit time and per railcar for demand $m$. \\
$\psi_{m}$ & Tardiness penalty cost per unit time and per railcar for demand $m$. \\
$\eta_{c}$ & Holding penalty cost per unit time and per railcar at yard $c$. \\
$h_{m}$ & Number of hazmat railcars for demand $m$. \\
$h_{m}$ & Number of regular railcars for demand $m$. \\
$s_{\ell}$ & Maximum speed that a train can run on service leg $\ell$. \\
$R_{\text {max }}$ & Risk threshold that the railroad carrier can reach on each service leg at any time instant. \\
$n_{k}$ & Number of yards in itinerary $k$. \\
$\alpha_{\ell k}^{i}$ & An indicator parameter which equals to 1 if service leg $\ell$ is the $i$ th leg in itinerary $k$, and 0 otherwise. \\
$\gamma_{c k}^{i}$ & An indicator parameter which equals to 1 if yard $c$ is the $i$ th yard in itinerary $k$, and 0 otherwise. \\
$R(\ell, s, t)$ & Risk incurred by trains on service leg $\ell$ at speed $s$ at time instant $t$. \\
\hline &
\end{tabular}

\subsection{Mathematical formulation}

In this section, we propose a mixed integer linear optimization model for the RTHD problem. We define the following non-negative decision variables:

- $x_{m k}$ is equal to 1 if demand $m$ is met using itinerary $k$, and 0 otherwise;

- $y_{m k s}^{i}$ is equal to 1 if the train runs at speed $s$ on $i$ th service leg in itinerary $k$ to meet demand $m$, and 0 otherwise; 
- $A_{m k}^{i}$ is the arrival time of trains at the $i$ th yard in itinerary $k$ to meet demand $m$, where $A_{m k}^{1}=0$ for all $m \in \mathcal{M}, k \in I^{m}$;

- $D_{m k}^{i}$ is the departure time of trains at the $i$ th yard in itinerary $k$ to meet demand $m$;

- $\Delta_{m k}^{i}$ is the idle time at the $i$ th yard in itinerary $k$ to meet demand $m$;

- $D_{m \ell}$ is the starting time of trains running on leg $\ell$ to meet demand $m$;

- $A_{m \ell}$ is the finish time of trains running on leg $\ell$ to meet demand $m$;

- $F_{m}$ is the finish time for meeting demand $m$;

- $E_{m}$ is the earliness of demand $m$, i.e. $E_{m}=\max \left\{d_{m}-F_{m}, 0\right\}$;

- $T_{m}$ is the tardiness of demand $m$, i.e. $T_{m}=\max \left\{F_{m}-d_{m}, 0\right\}$;

- $H_{\ell s t}$ is the total number of hazmat railcars that run on leg $\ell$ at speed $s$ at time $t$;

- $u_{m \ell t}$ is equal to 1 if the starting time of the train that run on leg $\ell$ to meet demand $m$ is greater than or equal to time instant $t$ (in other words, $D_{m \ell} \geq t$ ), and 0 otherwise;

- $v_{m \ell t}$ is equal to 1 if the starting time of the train that run on leg $\ell$ to meet demand $m$ is less than or equal to time instant $t$ (in other words, $D_{m \ell} \leq t$ ), and 0 otherwise;

- $w_{m \ell t}$ is equal to 1 if the starting time of the train that run on leg $\ell$ to meet demand $m$ is time instant $t$ (in other words, if $u_{m \ell t}=1$ and $v_{m \ell t}=1$, that is, $D_{m \ell}=t$ ), and 0 otherwise;

- $\delta_{m \ell s}$ is equal to 1 if the train runs on leg $\ell$ at speed $s$ to meet demand $m$, and 0 otherwise;

- $\sigma_{m \ell s t}$ is equal to 1 if the starting time of the train that run on leg $\ell$ to meet demand $m$ is time instant $t$ and its speed is $s$ (in other words, if $w_{m \ell t}=1$ and $\delta_{m \ell s}=1$ ), and 0 otherwise.

We denote $\hat{h}_{m}=h_{m}+\bar{h}_{m}$ as the total number of railcars required for each demand $m$. Let $K$ be a sufficient large number, then the RTHD problem can be modeled as follows: 


$$
\begin{aligned}
& \min \underbrace{\sum_{m \in \mathcal{M}} \sum_{k \in I^{m}} \sum_{i=1}^{n_{k}} \sum_{c \in \mathcal{C}} \hat{h}_{m} \eta_{c} \gamma_{c k}^{i} \Delta_{m k}^{i}}_{\text {Holding Cost }}+\underbrace{\sum_{m \in \mathcal{M}} \hat{h}_{m}\left(\theta_{m} E_{m}+\psi_{m} T_{m}\right)}_{\text {Earliness and Tardiness Costs }} \\
& \text { s.t. } A_{m k}^{i}=D_{m k}^{i-1}+\sum_{s \in \mathcal{S}} \sum_{\ell \in \mathcal{L}} p_{\ell s} \alpha_{\ell k}^{i-1} y_{m k s}^{i-1} \quad \text { for all } m \in \mathcal{M}, k \in I^{m}, i=2, \ldots, n_{k} \text {; } \\
& D_{m k}^{i}=A_{m k}^{i}+\Delta_{m k}^{i}+\sum_{c \in \mathcal{C}} q_{c} \gamma_{c k}^{i} x_{m k} \quad \text { for all } m \in \mathcal{M}, k \in I^{m}, i=1, \ldots, n_{k} \text {; } \\
& D_{m \ell}=\sum_{k \in I^{m}} \sum_{i=1}^{n_{k}-1} \alpha_{\ell k}^{i} D_{m k}^{i} \quad \text { for all } m \in \mathcal{M}, \ell \in \mathcal{L} ; \\
& A_{m \ell}=\sum_{k \in I^{m}} \sum_{i=2}^{n_{k}} \alpha_{\ell k}^{i-1} A_{m k}^{i} \quad \text { for all } m \in \mathcal{M}, \ell \in \mathcal{L} \\
& \delta_{m \ell s}=\sum_{k \in I^{m}} \sum_{i=1}^{n_{k}-1} \alpha_{\ell k}^{i} y_{m k s}^{i} \quad \text { for all } m \in \mathcal{M}, \ell \in \mathcal{L}, s \in \mathcal{S} ; \\
& \sum_{s \in \mathcal{S}} y_{m k s}^{i}=x_{m k} \quad \text { for all } m \in \mathcal{M}, k \in I^{m}, i=1, \ldots, n_{k}-1 \text {; } \\
& \sum_{k \in I^{m}} x_{m k}=1 \text { for all } m \in \mathcal{M} \\
& \sum_{s \in \mathcal{S}} s \delta_{m \ell s} \leq s_{\ell} \quad \text { for all } m \in \mathcal{M}, \ell \in \mathcal{L} \\
& D_{m k}^{n_{k}} \leq F_{m} \quad \text { for all } m \in \mathcal{M}, k \in I^{m} \text {; } \\
& F_{m}-D_{m k}^{n_{k}} \leq K\left(1-x_{m k}\right) \quad \text { for all } m \in \mathcal{M}, k \in I^{m} \text {; } \\
& D_{m \ell}-t \leq K u_{m \ell t}-1 \text { for all } m \in \mathcal{M}, \ell \in \mathcal{L}, t \in \mathcal{T} \text {; } \\
& t-D_{m \ell} \leq K\left(1-u_{m \ell t}\right) \quad \text { for all } m \in \mathcal{M}, \ell \in \mathcal{L}, t \in \mathcal{T} \text {; } \\
& D_{m \ell}-t \leq K\left(1-v_{m \ell t}\right) \quad \text { for all } m \in \mathcal{M}, \ell \in \mathcal{L}, t \in \mathcal{T} \text {; } \\
& t-D_{m \ell} \leq K v_{m \ell t}-1 \quad \text { for all } m \in \mathcal{M}, \ell \in \mathcal{L}, t \in \mathcal{T} \text {; } \\
& u_{m \ell t}+v_{m \ell t} \leq 1+w_{m \ell t} \quad \text { for all } m \in \mathcal{M}, \ell \in \mathcal{L}, t \in \mathcal{T} \text {; } \\
& w_{m \ell t} \leq u_{m \ell t} \quad \text { for all } m \in \mathcal{M}, \ell \in \mathcal{L}, t \in \mathcal{T} \text {; } \\
& w_{m \ell t} \leq v_{m \ell t} \quad \text { for all } m \in \mathcal{M}, \ell \in \mathcal{L}, t \in \mathcal{T} \text {; } \\
& w_{m \ell t}+\delta_{m \ell s} \leq 1+\sigma_{m \ell s t} \quad \text { for all } m \in \mathcal{M}, \ell \in \mathcal{L}, s \in \mathcal{S}, t \in \mathcal{T} \text {; } \\
& \sigma_{m \ell s t} \leq w_{m \ell t} \quad \text { for all } m \in \mathcal{M}, \ell \in \mathcal{L}, s \in \mathcal{S}, t \in \mathcal{T} \text {; }
\end{aligned}
$$




$$
\begin{aligned}
& \sigma_{m \ell s t} \leq \delta_{m \ell s} \quad \text { for all } m \in \mathcal{M}, \ell \in \mathcal{L}, s \in \mathcal{S}, t \in \mathcal{T} \\
& H_{\ell s t}=\sum_{m \in \mathcal{M}} h_{m} \sigma_{m \ell s t} \quad \text { for all } \ell \in \mathcal{L}, s \in \mathcal{S}, t \in \mathcal{T} \\
& r_{\ell s} H_{\ell s t} \leq R_{\max } \quad \text { for all } \ell \in \mathcal{L}, s \in \mathcal{S}, t \in \mathcal{T} \\
& x_{m k}, y_{m k s}^{i}, u_{m \ell t}, v_{m \ell t}, w_{m \ell s}, \delta_{m \ell t}, \sigma_{m \ell s t} \in\{0,1\} \\
& \quad \text { for all } m \in \mathcal{M}, k \in I^{m}, \ell \in \mathcal{L}, s \in \mathcal{S}, t \in \mathcal{T}, i=1, \ldots, n_{k}-1
\end{aligned}
$$

Objective (2a) is to minimize the total earliness and tardiness costs plus the holding cost. Constraints (2b) ensure that the arrival time of a train at a yard is its departure time at the previous yard plus its traveling time on the service leg that connects these two yards. Constraints (2c) ensure that the departure time of a train at a yard is its arrival time plus the idle time and the processing time at this yard. Constraints $(2 \mathrm{~d})$ and $(2 \mathrm{e})$ define the starting and finish time of a train on each service leg for each demand respectively. Constraints $(2 \mathrm{f})$ ensure that the variable $\delta$ takes its intended value. Constraints $(2 \mathrm{~g})-(2 \mathrm{~h})$ ensure that each demand is met by exactly one of its available itineraries. Constraints (2i) ensure that a train on a service leg can only run at a speed no greater than its maximum allowed speed. Constraints $(2 \mathrm{j})-(2 \mathrm{k})$ determine the finish time of each demand. Constraints (2l)- $(2 \mathrm{u})$ ensure that the variables $u, v, w, \sigma$ take their intended values, which enable us to keep track of the starting time of trains on each service leg for different demands. Constraints (2v) determine the total number of hazmat railcars that departure on each service leg at each speed at any time instant. Constraints $(2 \mathrm{w})$ ensure that the total risk incurred on each service leg at each speed at any time instant is no greater than the given threshold.

Note that in our model (2), derived from the eigth assumption, we actually assume that when more than one train departs the same service leg simultaneously at the same speed, the railcars of these trains are viewed to be consolidated in a single longer train. In reality, there may exist additional constraints on the maximum number of railcars that a train can have with some other operational requirements. Suppose there is a maximum number of railcars $U_{\ell}$ that a train can carry on service leg $\ell$, to tackle with this issue, we can extend our model by introducing the following constriants:

$$
\begin{array}{r}
H_{\ell s t}+\bar{H}_{\ell s t} \leq N_{\ell s t} U_{\ell}, \text { for all } \ell \in \mathcal{L}, s \in \mathcal{S}, t \in \mathcal{T} \\
H_{\ell s t}+\bar{H}_{\ell s t} \geq\left(N_{\ell s t}-1\right) U_{\ell}, \text { for all } \ell \in \mathcal{L}, s \in \mathcal{S}, t \in \mathcal{T}
\end{array}
$$

where $N_{\ell s t}$ is the number of trains that run on leg $\ell$ at speed $s$ at time $t$, and $\bar{H}_{\ell s t}$ is the 
total number of regular railcars that run on leg $\ell$ at speed $s$ at time $t$. By introducing these new variables and the corresponding constraints, we are able to consider further restrictions on the limit for the composition of a consolidated train. In this work, since we do not consider the cost related to the number of trains that are used, for the sake of simplicity, we will not consider such issue in the remainning of this paper.

\section{A lower bounding scheme for the RTHD problem}

Based on a reduction from the Minimum Weighted Sum Bin Packing problem (Epstein and Levin, 2008), we show that the RTHD problem is strongly NP-hard. For a complete proof, see Appendix. Given its NP-hardness, we propose a lower bounding scheme for the RTHD problem.

We first consider a variant of the RTHD problem, in which there is only one demand $m$. We refer to this problem as the $\operatorname{RTHD}(m)$ problem. Depending on the given risk threshold $R_{\max }$ and the maximum speed $s_{\ell}$ limited by the track quality, one can determine the set of available speeds $\mathcal{S}_{m \ell}$ on each service leg $\ell$ for demand $m$, that is,

$$
\mathcal{S}_{m \ell}=\left\{s \in \mathcal{S}: s \leq s_{\ell}, h_{m} r_{\ell s} \leq R_{\max }\right\}
$$

As we mentioned earlier, an itinerary can be viewed as a sequence of the yard and service leg operations. Suppose itinerary $k$ is selected to meet demand $m$. Then once we fix the idle time at each yard (i.e. $\Delta_{m k}^{i}$ ) and the speed at which trains run on each service leg (i.e. $y_{m k s}^{i}$ ), we get a specific routing and scheduling plan for meeting demand $m$ in itinerary $k$. Let $\mathcal{C}_{k}$ be the set of yards that a train passes in itinerary $k$. For the sake of simplicity, we assume that yard $c^{*}$ has the smallest holding penalty cost per unit time and per railcar in itinerary $k$, that is, $\eta_{c^{*}} \leq \eta_{c}$, for any $c \in \mathcal{C}_{k}$ (when there exists multiple yards that have smallest value of $\eta_{c}$, choose an arbitrary one as $\left.c^{*}\right)$. In addition, we denote the index of yard $c^{*}$ in itinerary $k$ as $i^{*}$.

Let $\lambda_{m k}$ denote a routing and scheduling plan for meeting demand $m$ in itinerary $k$, in which there is no idle time at any yard, that is,

$$
\lambda_{m k}=\left(y_{m k s_{1}}^{1}, \ldots, y_{m k s_{d}}^{1}, \ldots, y_{m k s_{1}}^{n_{k}-1}, \ldots, y_{m k s_{d}}^{n_{k}-1}\right) .
$$

Also, we denote $\mu_{m k}$ as the corresponding routing and scheduling plan generated from $\lambda_{m k}$ 
by assigning additional idle time at yards, that is,

$$
\mu_{m k}=\left(\Delta_{m k}^{1}, \ldots, \Delta_{m k}^{n_{k}}, \lambda_{m k}\right)
$$

Let $F\left(\lambda_{m k}\right)$ and $F\left(\mu_{m k}\right)$ be the finish time and $\operatorname{Cost}\left(\lambda_{m k}\right)$ and $\operatorname{Cost}\left(\mu_{m k}\right)$ be the total cost generated by the routing and scheduling plans $\lambda_{m k}$ and $\mu_{m k}$ respectively. Given any feasible plan $\lambda_{m k}$, we define $\mu_{m k}^{*}$ as the best plan generated from $\lambda_{m k}$ with a minimal total cost, that is, $\operatorname{Cost}\left(\mu_{m k}^{*}\right) \leq \operatorname{Cost}\left(\mu_{m k}\right)$, for any $\mu_{m k}$ generated from $\lambda_{m k}$.

The following lemmas describe some structural properties of optimal routing and scheduling plans for the $\operatorname{RTHD}(m)$ problem.

Lemma 5.1. Suppose $\mu_{m k}=\left(0, \ldots, \Delta_{m k}^{i^{*}}=\rho, \ldots, 0, \lambda_{m k}\right)$ and $\mu_{m k}^{\prime}=\left(0, \ldots, \Delta_{m k}^{j}=\right.$ $\left.\rho, \ldots, 0, \lambda_{m k}\right)$ are two plans generated from $\lambda_{m k}$, then we have $\operatorname{Cost}\left(\mu_{m k}\right) \leq \operatorname{Cost}\left(\mu_{m k}^{\prime}\right)$.

Proof. It is easy to know that $F\left(\mu_{m k}\right)=F\left(\mu_{m k}^{\prime}\right)$. So the earliness and tardiness costs for both plans are the same. Recall that the $i^{*}$ th yard is $c^{*}$, as a result, we have $\operatorname{Cost}\left(\mu_{m k}\right)-$ $\operatorname{Cost}\left(\mu_{m k}^{\prime}\right)=\hat{h}_{m} \rho\left(\eta_{c^{*}}-\eta_{c}\right) \leq 0$, where $c$ is the $j$ th yard in itinerary $k$.

Corollary 5.1. If $\mu_{m k}^{*}$ has idle time at yards, then the idle time can be assigned to only yard $c^{*}$.

Lemma 5.2. If $F\left(\lambda_{m k}\right)>d_{m}$, then $\mu_{m k}^{*}=\left(0, \ldots, 0, \lambda_{m k}\right)$.

Proof. It is easy to know that $\operatorname{Cost}\left(\lambda_{m k}\right)=\hat{h}_{m} \psi_{m}\left(F\left(\lambda_{m k}\right)-d_{m}\right)$. Suppose we add $\rho$ idle time at yard $c^{*}$, that is, $\mu_{m k}=\left(0, \ldots, \Delta_{m k}^{i^{*}}=\rho, \ldots, 0, \lambda_{m k}\right)$. Then $\operatorname{Cost}\left(\mu_{m k}\right)=$ $\hat{h}_{m}\left(\rho \eta_{c^{*}}+\psi_{m}\left(F\left(\lambda_{m k}\right)-d_{m}+\rho\right)\right)$. Note that

$$
\operatorname{Cost}\left(\mu_{m k}\right)-\operatorname{Cost}\left(\lambda_{m k}\right)=\hat{h}_{m} \rho\left(\eta_{c^{*}}+\psi_{m}\right)>0,
$$

we know that $\mu_{m k}^{*}=\left(0, \ldots, 0, \lambda_{m k}\right)$.

Lemma 5.3. If $F\left(\lambda_{m k}\right)<d_{m}$, then we have

(a) when $\eta_{c^{*}} \leq \theta_{m}, \mu_{m k}^{*}=\left(0, \ldots, \Delta_{m k}^{i^{*}}=d_{m}-F\left(\lambda_{m k}\right), \ldots, 0, \lambda_{m k}\right)$.

(b) when $\eta_{c^{*}}>\theta_{m}, \mu_{m k}^{*}=\left(0, \ldots, 0, \lambda_{m k}\right)$.

Proof. The total cost for $\lambda_{m k}$ is $\operatorname{Cost}\left(\lambda_{m k}\right)=\hat{h}_{m} \theta_{m}\left(d_{m}-F\left(\lambda_{m k}\right)\right)$. Now we add $\rho$ idle time at yard $c^{*}$, i.e. $\mu_{m k}=\left(0, \ldots, \Delta_{m k}^{i^{*}}=\rho, \ldots, 0, \lambda_{m k}\right)$. From Lemma 5.2, we must 
have $\rho \leq d_{m}-F\left(\lambda_{m k}\right)$; otherwise, additional tardiness cost will be incurred. Note that $\operatorname{Cost}\left(\mu_{m k}\right)=\hat{h}_{m}\left(\theta_{m}\left(d_{m}-F\left(\lambda_{m k}\right)-\rho\right)+\rho \eta_{c^{*}}\right)$, so we have

$$
\operatorname{Cost}\left(\mu_{m k}\right)-\operatorname{Cost}\left(\lambda_{m k}\right)=\hat{h}_{m} \rho\left(\eta_{c^{*}}-\theta_{m}\right) .
$$

As a result, when $\eta_{c^{*}} \leq \theta_{m}$, we have $\rho=d_{m}-F\left(\lambda_{m k}\right)$; and when $\eta_{c^{*}}>\theta_{m}$, we have $\rho=0$.

Lemma 5.4. Suppose $\lambda_{m k}$ and $\lambda_{m k}^{\prime}$ are two feasible plans to the RTHD(m) problem. If $F\left(\lambda_{m k}\right) \leq F\left(\lambda_{m k}^{\prime}\right) \leq d_{m}$, then $\operatorname{Cost}\left(\mu_{m k}^{\prime *}\right) \leq \operatorname{Cost}\left(\mu_{m k}^{*}\right)$, where $\mu_{m k}^{\prime *}$ and $\mu_{m k}^{*}$ are the best plans generated from $\lambda_{m k}^{\prime}$ and $\lambda_{m k}$ respectively.

Proof. Note that $\operatorname{Cost}\left(\lambda_{m k}^{\prime}\right)=\hat{h}_{m} \theta_{m}\left(d_{m}-F\left(\lambda_{m k}^{\prime}\right)\right)$ and $\operatorname{Cost}\left(\lambda_{m k}\right)=\hat{h}_{m} \theta_{m}\left(d_{m}-F\left(\lambda_{m k}\right)\right)$. From Lemma 5.3, when $\eta_{c^{*}} \leq \theta_{m}$, we have $\operatorname{Cost}\left(\mu_{m k}^{\prime *}\right)=\hat{h}_{m} \eta_{c^{*}}\left(d_{m}-F\left(\lambda_{m k}^{\prime}\right)\right)$ and $\operatorname{Cost}\left(\mu_{m k}^{*}\right)=\hat{h}_{m} \eta_{c^{*}}\left(d_{m}-F\left(\lambda_{m k}\right)\right)$. So we have

$$
\operatorname{Cost}\left(\mu_{m k}^{*}\right)-\operatorname{Cost}\left(\mu_{m k}^{*}\right)=\hat{h}_{m} \eta_{c^{*}}\left(F\left(\lambda_{m k}\right)-F\left(\lambda_{m k}^{\prime}\right)\right) \leq 0 ;
$$

when $\eta_{c^{*}}>\theta_{m}$, we have $\operatorname{Cost}\left(\mu_{m k}^{\prime *}\right)=\operatorname{Cost}\left(\lambda_{m k}^{\prime}\right)$ and $\operatorname{Cost}\left(\mu_{m k}^{*}\right)=\operatorname{Cost}\left(\lambda_{m k}\right)$, so we have $\operatorname{Cost}\left(\mu_{m k}^{\prime *}\right)-\operatorname{Cost}\left(\mu_{m k}^{*}\right)=\hat{h}_{m} \theta_{m}\left(F\left(\lambda_{m k}\right)-F\left(\lambda_{m k}^{\prime}\right)\right) \leq 0$.

Lemma 5.5. Suppose $\lambda_{m k}$ and $\lambda_{m k}^{\prime}$ are two feasible plans to the RTHD(m) problem. If $F\left(\lambda_{m k}\right) \geq F\left(\lambda_{m k}^{\prime}\right) \geq d_{m}$, then $\operatorname{Cost}\left(\mu_{m k}^{\prime *}\right) \leq \operatorname{Cost}\left(\mu_{m k}^{*}\right)$, where $\mu_{m k}^{\prime *}$ and $\mu_{m k}^{*}$ are the best plans generated from $\lambda_{m k}^{\prime}$ and $\lambda_{m k}$ respectively.

Proof. Note that $\operatorname{Cost}\left(\lambda_{m k}^{\prime}\right)=\hat{h}_{m} \psi_{m}\left(F\left(\lambda_{m k}^{\prime}\right)-d_{m}\right)$ and $\operatorname{Cost}\left(\lambda_{m k}\right)=\hat{h}_{m} \psi_{m}\left(F\left(\lambda_{m k}\right)-d_{m}\right)$. From Lemma 5.2, we know that $\operatorname{Cost}\left(\mu_{m k}^{\prime *}\right)=\operatorname{Cost}\left(\lambda_{m k}^{\prime}\right)$, and $\operatorname{Cost}\left(\mu_{m k}^{*}\right)=\operatorname{Cost}\left(\lambda_{m k}\right)$, so we have

$$
\operatorname{Cost}\left(\mu_{m k}^{*}\right)-\operatorname{Cost}\left(\mu_{m k}^{*}\right)=\hat{h}_{m} \psi_{m}\left(F\left(\lambda_{m k}^{\prime}\right)-F\left(\lambda_{m k}\right)\right) \leq 0,
$$

and the lemma follows.

Let $\Lambda_{m k}^{-}=\left\{\lambda_{m k}: F\left(\lambda_{m k}\right) \leq d_{m}\right\}$ and $\Lambda_{m k}^{+}=\left\{\lambda_{m k}: F\left(\lambda_{m k}\right) \geq d_{m}\right\}$. We define $\lambda_{m k}^{-} \in \Lambda_{m k}^{-}$and $\lambda_{m k}^{+} \in \Lambda_{m k}^{+}$be the best plans that have the minimal cost in $\Lambda_{m k}^{-}$and $\Lambda_{m k}^{+}$ respectively. From Lemmas 5.4 and 5.5, we know that $F\left(\lambda_{m k}\right) \leq F\left(\lambda_{m k}^{-}\right) \leq d_{m}$ for any $\lambda_{m k} \in \Lambda_{m k}^{-}$, and $F\left(\lambda_{m k}\right) \geq F\left(\lambda_{m k}^{+}\right) \geq d_{m}$ for any $\lambda_{m k} \in \Lambda_{m k}^{+}$. Let $\mu_{m k}^{o p t}$ be the optimal plan to the $\operatorname{RTHD}(m)$ problem that meets demand $m$ by itinerary $k$. Then we have the following theorem. 
Theorem 5.1. $\mu_{m k}^{o p t}$ can be calculated as follows:

- Case 1: $\eta_{c^{*}} \leq \theta_{m}$. If $\eta_{c^{*}}\left(d_{m}-F\left(\lambda_{m k}^{-}\right)\right) \leq \psi_{m}\left(F\left(\lambda_{m k}^{+}\right)-d_{m}\right)$, then $\mu_{m k}^{o p t}=\left(0, \ldots, \Delta_{m k}^{i^{*}}=\right.$ $\left.d_{m}-F\left(\lambda_{m k}^{-}\right), \ldots, 0, \lambda_{m k}^{-}\right) ;$else, $\mu_{m k}^{o p t}=\left(0, \ldots, 0, \lambda_{m k}^{+}\right)$.

- Case 2: $\eta_{c^{*}}>\theta_{m}$. If $\theta_{m}\left(d_{m}-F\left(\lambda_{m k}^{-}\right)\right) \leq \psi_{m}\left(F\left(\lambda_{m k}^{+}\right)-d_{m}\right)$, then $\mu_{m k}^{o p t}=\left(0, \ldots, 0, \lambda_{m k}^{-}\right)$; else, $\mu_{m k}^{o p t}=\left(0, \ldots, 0, \lambda_{m k}^{+}\right)$.

Proof. The theorem follows directly from Lemmas 5.1 to 5.5.

Given any itinerary $k$ to meet demand $m$, the problem of calculating $\lambda_{m k}^{-}$and $\lambda_{m k}^{+}$can be described as follows: Given $X_{i}=\left(p_{\ell_{i} s_{1}}, p_{\ell_{i} s_{2}}, \ldots, p_{\ell_{i} s_{d}}\right)$ for $i=1, \ldots, n_{k}-1$, find the elements in the multiset $\sum_{i=1}^{n_{k}-1} X_{i}=\left\{\sum_{i=1}^{n_{k}-1} x_{i} \mid x_{i} \in X_{i}\right\}$ that are nearest to the value of $d_{m}-\sum_{c \in \mathcal{C}} \sum_{i=1}^{n_{k}} q_{c} \gamma_{c k}^{i}$ from the left and the right sides, respectively. Note that such problem is a variant of the $K$ th LARGEST $m$-TUPLE problem, which is not known to be in NP (Johnson and Mizoguchi, 1978; Garey and Johnson, 1979). In the work by Johnson and Mizoguchi (1978), the authors proposed a pseudo-polynomial time algorithm for this problem. In particular, when $n_{k}$ is fixed, they showed that the problem can be solved in polynomial time.

In real-life rail transportations, the number of available speeds and the number of service legs in an itinerary are generally within a small range. So we construct the following integer programs to calculate $\lambda_{m k}^{-}$(Problem P1) and $\lambda_{m k}^{+}$(Problem P2), which can be solved quickly by optimization solvers (e.g. Gurobi or CPLEX) for small and medium instances.

Problem P1 is as follows:

$$
\begin{gathered}
\min \quad d_{m}-\sum_{c \in \mathcal{C}} \sum_{i=1}^{n_{k}} q_{c} \gamma_{c k}^{i}-\sum_{l \in \mathcal{L}} \sum_{i=1}^{n_{k}-1} \sum_{s \in \mathcal{S}_{m \ell}} p_{\ell s} \alpha_{\ell k}^{i} y_{m k s}^{i} \\
\sum_{l \in \mathcal{L}} \sum_{s \in \mathcal{S}_{m \ell}} \sum_{i=1}^{n_{k}-1} p_{\ell s} \alpha_{\ell k}^{i} y_{m k s}^{i} \leq d_{m}-\sum_{c \in \mathcal{C}} \sum_{i=1}^{n_{k}} q_{c} \gamma_{c k}^{i} \\
\sum_{s \in \mathcal{S}_{m \ell}} y_{m k s}^{i}=1 \quad \text { for } i=1, \ldots, n_{k}-1 \\
y_{m k s}^{i} \in\{0,1\} \quad \text { for } s \in \mathcal{S}_{m \ell}, i=1, \ldots, n_{k}-1
\end{gathered}
$$

Problem P2 is as follows:

$$
\min \sum_{l \in \mathcal{L}} \sum_{i=1}^{n_{k}-1} \sum_{s \in \mathcal{S}_{m \ell}} p_{\ell s} \alpha_{\ell k}^{i} y_{m k s}^{i}+\sum_{c \in \mathcal{C}} \sum_{i=1}^{n_{k}} q_{c} \gamma_{c k}^{i}-d_{m}
$$




$$
\begin{aligned}
& \sum_{l \in \mathcal{L}} \sum_{s \in \mathcal{S}_{m \ell}} \sum_{i=1}^{n_{k}-1} p_{\ell s} \alpha_{\ell k}^{i} y_{m k s}^{i} \geq d_{m}-\sum_{c \in \mathcal{C}} \sum_{i=1}^{n_{k}} q_{c} \gamma_{c k}^{i} ; \\
& \sum_{s \in \mathcal{S}_{m \ell}} y_{m k s}^{i}=1 \quad \text { for } i=1, \ldots, n_{k}-1 ; \\
& y_{m k s}^{i} \in\{0,1\} \quad \text { for } s \in \mathcal{S}_{m \ell}, i=1, \ldots, n_{k}-1
\end{aligned}
$$

From our fourth assumption on Page 8, we know that at least one feasible routing and scheduling plan for each demand always exists, that is, for each demand there must exist a feasible plan with a finish time either earlier than, equal to, or later than its due date. As a result, there must exist at least a feasible solution for either Problem P1 or Problem P2. Based on the solutions from Problems P1 and P2, we are able to construct an algorithm for the $\operatorname{RTHD}(m)$ problem (see Algorithm 5.1) and also a lower bounding scheme for the general RTHD problem (see Algorithm 5.2). For simplicity, we denote Algorithm 5.2 as Algorithm LB, and the cost derived from this algorithm as Cost(LB).

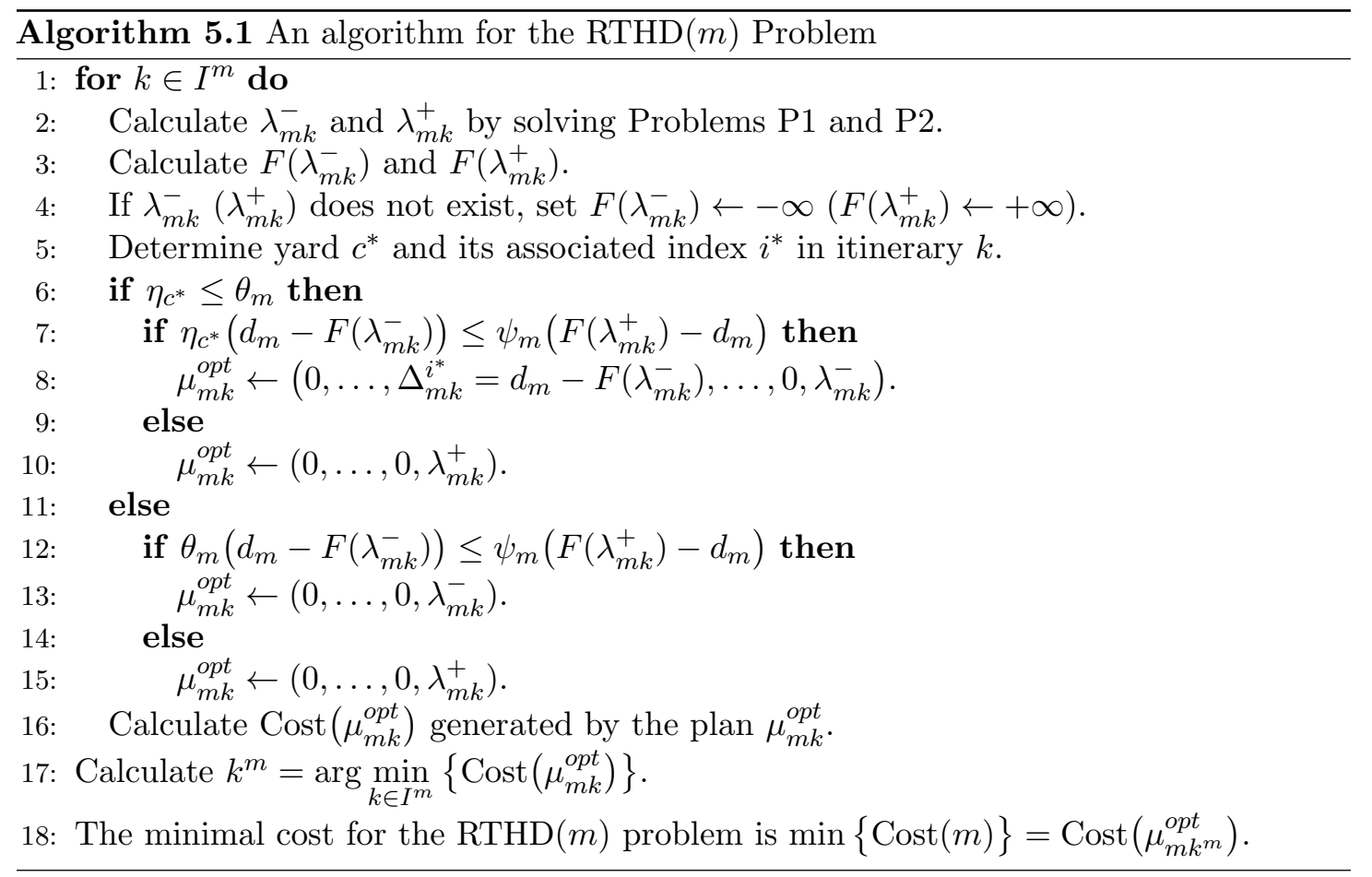




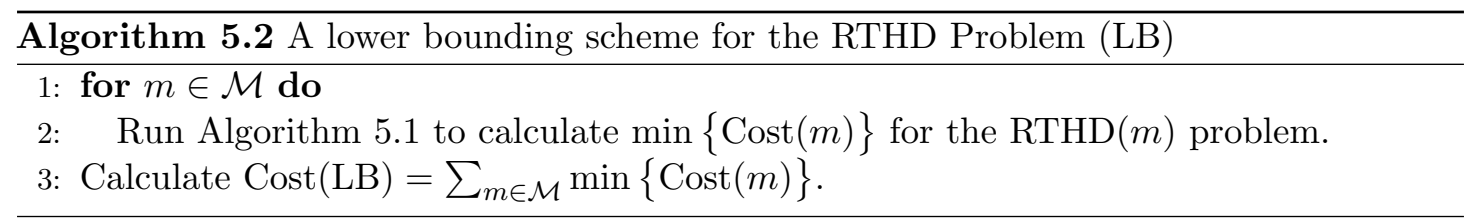

\section{A time-and-service-leg-based heuristic algorithm for the RTHD problem}

From Algorithm 5.1, we are able to obtain the optimal routing and scheduling plan $\mu_{m k^{m}}^{o p t}$ for each $\operatorname{RTHD}(m)$ problem, where $k^{m}=\arg \min _{k \in I^{m}}\left\{\operatorname{Cost}\left(\mu_{m k}^{o p t}\right)\right\}$. In this section, we propose a heuristic algorithm to find feasible plans to the RTHD problem. The main idea of our heuristic algorithm is as follows. For each demand $m$, we first run Algorithm 5.1 to calculate $\mu_{m k^{m}}^{o p t}$. Then, we select the corresponding itinerary $k^{m}$ to meet demand $m$, and fix the speeds of trains on each service leg $s_{m \ell}$ in itinerary $k^{m}$ which are determined by $\mu_{m k^{m}}^{o p t}$. Note that such routing and scheduling plan may not be a feasible plan for the general RTHD problem due to the potential violation of the imposed risk threshold, which makes constraints $(2 \mathrm{w})$ infeasible. So we need to modify the above plan once the risk threshold is violated, by adding additional idle time at yards for some demands until the modified plan becomes feasible.

In order to determine which demands should be delayed at yards, we define $\Omega_{m c}$ as the increment of total cost when we impose one unit of additional idle time at any yard $c \in \mathcal{C}_{k^{m}}$ for meeting demand $m$. From Theorem 5.1 we know that there are three possible scenarios of the plan $\mu_{m k^{m}}^{o p t}$ :

- Case 1: $\mu_{m k^{m}}^{o p t}=\left(0, \ldots, \Delta_{m k^{*}}^{i^{*}}=d_{m}-F\left(\lambda_{m k^{m}}^{-}\right), \ldots, 0, \lambda_{m k^{m}}^{-}\right)$. In this case, we have $\Omega_{m c}=\hat{h}_{m}\left(\eta_{c}+\psi_{m}\right)$.

- Case 2: $\mu_{m k^{m}}^{o p t}=\left(0, \ldots, 0, \lambda_{m k^{m}}^{-}\right)$. In this case, we have $\Omega_{m c}=\hat{h}_{m}\left(\eta_{c}-\theta_{m}\right)$.

- Case 3: $\mu_{m k^{m}}^{o p t}=\left(0, \ldots, 0, \lambda_{m k^{m}}^{+}\right)$. In this case, we have $\Omega_{m c}=\hat{h}_{m}\left(\eta_{c}+\psi_{m}\right)$.

We propose a time-and-service-leg-based heuristic algorithm to delay specific demands according to the values of $\Omega_{m c}$, so as to determine the feasible routing and scheduling plans. For each $(\ell, s, t)$ triple, we first determine the set of demands that trains start to travel at time instant $t$ on service leg $\ell$ at speed $s$. For illustration purpose, we denote such set of demands as $D(\ell, s, t)$. Suppose the origin yard for service leg $\ell$ is yard $c$, then we sort $D(\ell, s, t)$ according to nonincreasing order of $\Omega_{m c}$. If the risk threshold is violated for these demands at time instant $t$ on service leg $\ell$ at speed $s$, we then delay the departure 
time of certain demands in $D(\ell, s, t)$ to construct a feasible routing and scheduling plan, see Algorithm 6.1. For simplicity, we denote $\mathcal{E}$ as the set of time instances that will be examined during the heuristic algorithm. We denote Algorithm 6.1 as Algorithm TSLB, and its cost as Cost(TSLB).

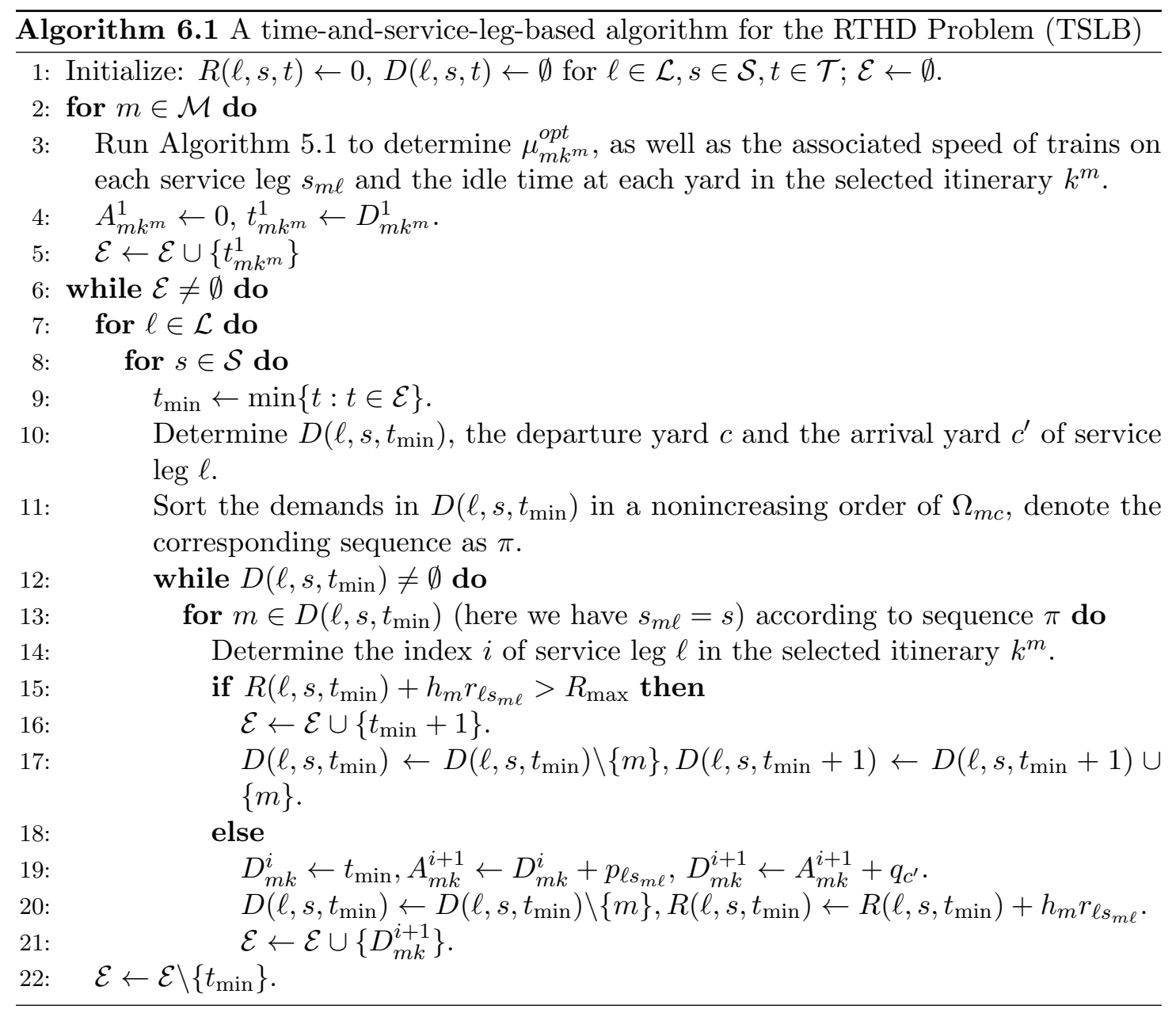

\section{Experimental study}

\subsection{Parameter estimation: service-leg traveling time and risk assessment}

In this section, we use the proposed lower bounding scheme and Algorithm TSLB to solve instances arising from real-life rail networks. Before that, we first provide the basic information about parameters which will be used in our experimental study. 
Consistent with literature (Verma, 2011), the risk posed by a hazmat railcar on a train that is traveling at speed $s$ on service leg $\ell$ is:

$$
r_{\ell s}=P(D \mid A) \times P(H \mid D, A) \times P(R \mid D, H, A) \times P E_{\ell},
$$

where $P(H \mid D, A)$ is the probability that a hazmat railcar derailed given a train accident $A$ occurs; $P(R \mid D, H, A)$ is the probability of release from a hazmat railcars given a train accident $A$ occurs; and $P E_{\ell}$ is the population exposure due to the release per hazmat railcar given a train accident $A$ occurs. From the FRA dataset, we know that there are 882 hazamt derailment accidents and 169 hazmat release accidents among all the 5,476 derailment accidents, so we obtain the approximate value of the above probabilities as $P(H \mid D, A)=882 / 5476=0.16$ and $P(R \mid D, H, A)=169 / 5476=0.03$. From Equation (5) and the relationship between the train speed and derailment rate obtained in Section 3, i.e. Equation (1), we can get

$$
r_{\ell s}=(0.0028 s+0.043) \times 0.0048 \times P E_{\ell},
$$

where $P E_{\ell}$ is the population exposure when one hazmat railcar traverses service leg $\ell$, which is calculated as follows. We focus on hazmat that become airborne in the event of an accidental release (such as chlorine, propane and ammonia) since they can travel long distances due to wind and expose large areas to health and environmental risks. Spatial distribution of toxic concentration level is estimated using Gaussian plume model (GPM), and at any given distance the maximum concentration is observed at the downwind locations (Arya, 1999). We use the immediately dangerous to life and health (IDLH) concentration levels of the hazmat being shipped in determining the threshold distances for fatality and injuries (http://www.cdc.gov/niosh). In estimating the population exposure, we adopt the worst-case approach outlined in Verma and Verter (2007) that assumes least favorable weather conditions and focusing on maximum concentrate levels. The proposed approach not only results in conservative evacuation distances, but also generates a danger circle that would carve the population exposure band along the service-leg $\ell$ (i.e., aggregate risk measure as proposed in Batta and Chiu (1988b) and ReVelle et al. (1991)). It is important that while the conservative evacuation distances does address the concern about wind speed, the generation of danger circle circumvents the limitations imposed by the absence of a priori information about wind direction. For expositional reasons, we do not repeat the methodological details and invite the reader to consult Verma and Verter (2007) for 
relevant steps. Note that the impact of uncertain wind direction and wind speed can also be addressed by using the technique proposed in Patel and Horowitz (1994), or in Sivakumar and Batta (1994).

\subsection{Computational environment}

In this section, we test the performance of our proposed mixed integer programming formulation and Algorithm TSLB. We use Gurobi Optimizer 6.5.1 and the Python programming language to code and solve all the mixed integer programs and algorithms on a computer with a $3.6 \mathrm{GHz}$ Intel-Core i7-4790 processor and $16 \mathrm{~GB}$ of RAM running the Linux operating system.

To conduct our experiments, we consider a rail network of Norfolk Southern (NS) in the Midwest United States, a Class I railroad operator, as shown in Figure 2. A total of 82 service legs are created in ArcView GIS (Arcview, 1996), connecting any two of the 25 yards in the network. This structure indicates 600 origin-destination pairs. In addition, according to the FRA database, there is no accident with a speed greater than $80 \mathrm{mph}$ for the freight trains. As a result, it is reasonable to assume that in the network, the train can only run at a speed (mph) in the following discrete speed set: $\mathcal{S}=\{10,25,40,60,80\}$, depending on the types of track classes. For the sake of investigating the importance of train speeds, we randomly generate data for the track class of each service leg $\ell$ with a maximum allowed speed $s_{\ell} \in\{40,60,80\}$. Moreover, in order to limit the number of itineraries, we add a restriction that the number of intermediate handling for each demand should not be greater than four. Given the network structure, the number of itineraries for each original-destination pair ranges from 1 to 37, and the total number of itineraries for all these pairs is 6019 . We use hypothetical demand data, which were generated randomly. For each origin-destination pair, it is easy to know its smallest and largest delivery time according to the set of available speeds on each service leg for that demand in the network. For simplicity, for each origin-destination pair, we denote $\underline{L}$ and $\bar{L}$ as the smallest and largest delivery time respectively, and we call $\left[\underline{L}_{m}, \bar{L}_{m}\right]$ the delivery time range for demand $m$. We assume that the due date for each demand must be greater than its smallest delivery time $\underline{L}$, otherwise we would end up with an unreasonable solution. Note that in all of the following experiments each parameter related to the rail network is not random but realistic, except for the hypothetical demands and track classes. 


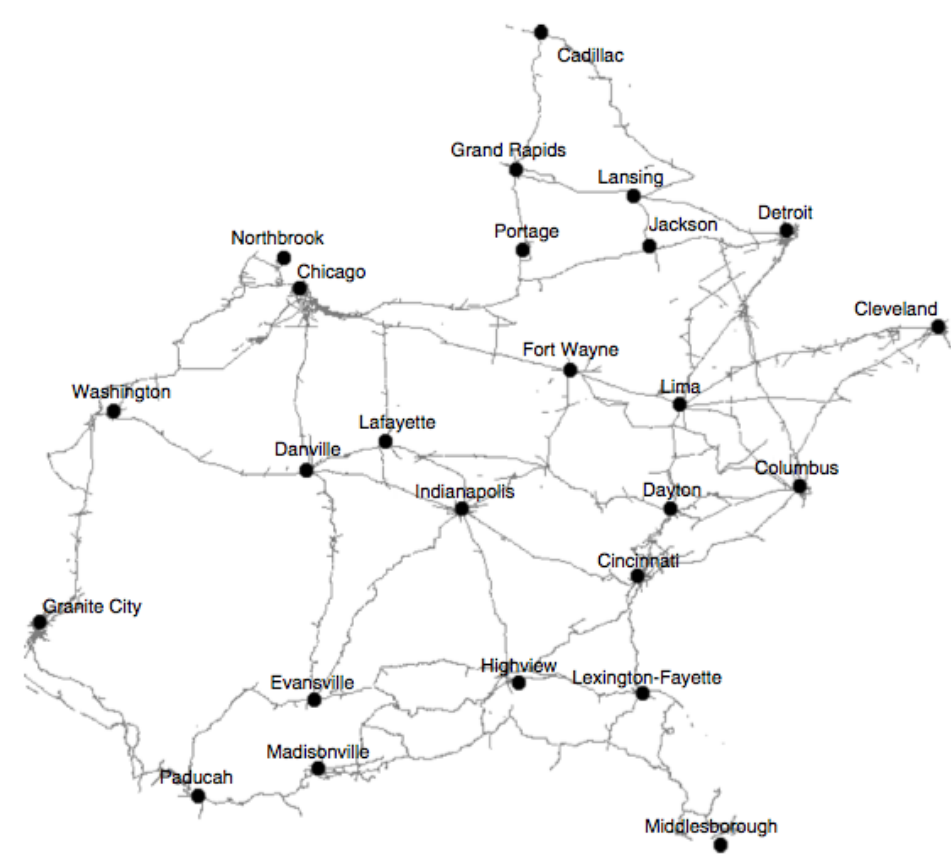

Figure 2: Rail network (Verma et al., 2011).

\subsection{Experiment 1: Performance of the MIP formulation}

\subsubsection{Trade-off between total cost and risk threshold}

From Section 7.1, we know that when a train runs at a higher speed, its traveling time on a service leg decreases, while the risk incurred by the train per hazmat railcar increases. Due to the restriction on risk threshold, the railcars for some of the demands may not be able to consolidate with other demands into a train at the same departure time at higher speed, even when it is possible for them to be consolidated at a lower speed. This fact results in delays for some of the demands. In other words, the higher the risk threshold, the more flexible shipment plans that we can obtain to reduce the costs. Based on this observation, one expects a trade-off between the total cost and risk threshold, especially when multiple demands share common service legs.

To illustrate the relationship between the total cost and risk threshold, we consider an example of an instance with 5 demands that have the same origin-destination pair (i.e. Evansville $\rightarrow$ Lima) in the given network. For this origin-destination pair, there are five available itineraries in the given rail network: 
- itinerary $k_{1}$ : Evansville $\stackrel{\ell_{1}}{\longrightarrow}$ Danville $\stackrel{\ell_{2}}{\longrightarrow}$ Indiannapolis $\stackrel{\ell_{3}}{\longrightarrow}$ Fort Wayne $\stackrel{\ell_{4}}{\longrightarrow}$ Lima;

- itinerary $k_{2}$ : Evansville $\stackrel{\ell_{5}}{\longrightarrow}$ Indiannapolis $\stackrel{\ell_{3}}{\longrightarrow}$ Fort Wayne $\stackrel{\ell_{6}}{\longrightarrow}$ Jackson $\stackrel{\ell_{7}}{\longrightarrow}$ Lima;

- itinerary $k_{3}$ : Evansville $\stackrel{\ell_{5}}{\longrightarrow}$ Indiannapolis $\stackrel{\ell_{3}}{\longrightarrow}$ Fort Wayne $\stackrel{\ell_{4}}{\longrightarrow}$ Lima;

- itinerary $k_{4}$ : Evansville $\stackrel{\ell_{5}}{\longrightarrow}$ Indiannapolis $\stackrel{\ell_{8}}{\longrightarrow}$ Lafayette $\stackrel{\ell_{9}}{\longrightarrow}$ Fort Wayne $\stackrel{\ell_{4}}{\longrightarrow}$ Lima;

- itinerary $k_{5}$ : Evansville $\stackrel{\ell_{5}}{\longrightarrow}$ Indiannapolis $\stackrel{\ell_{10}}{\longrightarrow}$ Cincinnati $\stackrel{\ell_{11}}{\longrightarrow}$ Dayton $\stackrel{\ell_{12}}{\longrightarrow}$ Lima.

The above itineraries include 12 service legs and 9 yards. The parameters for the service legs and yards are given in Tables 3 and 4 respectively, and the parameters for the demands are given in Table 5 .

Table 3: Parameters for the service legs

\begin{tabular}{c|c|c|c|c|c|c|c|c|c|c|c}
\hline \multirow{2}{*}{ Service leg } & \multicolumn{9}{|c|}{ traveling time (hours) } & \multicolumn{5}{c}{ risk $\left(\times 10^{-4}\right)$} \\
\cline { 2 - 11 } & $10 \mathrm{mph}$ & $25 \mathrm{mph}$ & $40 \mathrm{mph}$ & $60 \mathrm{mph}$ & $80 \mathrm{mph}$ & $10 \mathrm{mph}$ & $25 \mathrm{mph}$ & $40 \mathrm{mph}$ & $60 \mathrm{mph}$ & $80 \mathrm{mph}$ \\
\hline$\ell_{1}$ & 16 & 7 & 4 & 3 & 2 & 36 & 57 & 78 & 106 & 134 \\
$\ell_{2}$ & 10 & 4 & 3 & 2 & - & 79 & 126 & 172 & 234 & - \\
$\ell_{3}$ & 12 & 5 & 3 & 2 & 2 & 138 & 219 & 300 & 409 & 517 \\
$\ell_{4}$ & 7 & 3 & 2 & 2 & - & 91 & 145 & 198 & 270 & - \\
$\ell_{5}$ & 16 & 7 & 4 & 3 & 2 & 62 & 98 & 134 & 183 & 231 \\
$\ell_{6}$ & 18 & 7 & 5 & 3 & 3 & 27 & 42 & 58 & 78 & 99 \\
$\ell_{7}$ & 16 & 7 & 4 & 3 & 2 & 38 & 60 & 82 & 111 & 140 \\
$\ell_{8}$ & 7 & 3 & 2 & 2 & - & 154 & 245 & 335 & 456 & - \\
$\ell_{9}$ & 11 & 5 & 3 & 2 & 2 & 34 & 54 & 74 & 101 & 127 \\
$\ell_{10}$ & 11 & 5 & 3 & 2 & 2 & 98 & 156 & 213 & 290 & 367 \\
$\ell_{11}$ & 7 & 3 & 2 & 2 & - & 184 & 293 & 402 & 546 & - \\
$\ell_{12}$ & 10 & 4 & 3 & 2 & - & 72 & 115 & 157 & 214 & - \\
\hline
\end{tabular}

-: not allowed to run at this speed restricted by the track classes.

Table 4: Parameters for the yards

\begin{tabular}{r|c|c}
\hline Yard & Processing time $\left(q_{c}\right)$ & holding cost $\left(\eta_{c}\right)$ \\
\hline Evansville & 3 & 51 \\
Danville & 3 & 97 \\
Indiannapolis & 2 & 75 \\
Fort Wayne & 2 & 55 \\
Lima & 2 & 50 \\
Jackson & 3 & 78 \\
Lafayette & 2 & 53 \\
Cincinnati & 1 & 42 \\
Dayton & 3 & 94 \\
\hline
\end{tabular}

We further define the minimum risk threshold

$$
\underline{R}=\max _{m \in \mathcal{M}}\left\{\min _{k \in I^{m}}\left\{\max _{\ell \in \mathcal{L}}\left\{h_{m} r_{\ell 1}: \alpha_{\ell k}^{i}=1 \text { for some } i\right\}\right\}\right\}
$$


Table 5: Parameters for the demands

\begin{tabular}{c|c|c|c|c|c}
\hline Demand & hazmat $\left(h_{m}\right)$ & regular $\left(\bar{h}_{m}\right)$ & earliness $\left(\theta_{m}\right)$ & tardiness $\left(\psi_{m}\right)$ & due date $\left(d_{m}\right)$ \\
\hline 1 & 3 & 4 & 22 & 90 & 46 \\
2 & 3 & 4 & 93 & 89 & 72 \\
3 & 3 & 4 & 22 & 37 & 15 \\
4 & 3 & 4 & 42 & 49 & 31 \\
5 & 3 & 4 & 8 & 92 & 47 \\
\hline
\end{tabular}

and the maximum risk threshold

$$
\bar{R}=\max _{\ell \in \mathcal{L}}\left\{\sum_{m \in \mathcal{M}_{\ell}} h_{m} r_{\ell s_{d}}\right\}
$$

where

$$
\mathcal{M}_{\ell}=\left\{m \in \mathcal{M}: \exists k \in I^{m} \text { such that } \alpha_{\ell k}^{i}=1 \text { for some } i\right\} .
$$

Note that when $R_{\max } \leq \underline{R}$, there is no feasible routing and scheduling plan for the demands, and when $R_{\max } \geq \bar{R}$, the risk threshold has no effect on the routing and scheduling plan decisions. For simplicity, we call $[\underline{R}, \bar{R}]$ the risk threshold range.

Figure 3 depicts the different solutions obtained for the above instance, from which we can see that the cost savings are traded-off against risk threshold. Using the solutions obtained by solving the MIP, one can easily determine the efficient frontier and output the nondominated solutions in railroad carriers' final decision-making. For example, in Figure 3, the efficient frontier are those points listed in Table 6 (see the first row with cost $C_{1}$ ), while all the other points are the dominated solutions which the railroad carriers should avoid to choose.

On the other hand, the total system risk shows an overall upward trend as the risk threshold increases (Figure 4). But there is no strict trade-off between the risk threshold and total risk. For example, when the risk threshold increases from $7.93 \times 10^{-2}$ to $9.14 \times 10^{-2}$, the total risk increases from $64.29 \times 10^{-2}$ to $74.22 \times 10^{-2}$, while the total risk decreases from $74.22 \times 10^{-2}$ to $72.06 \times 10^{-2}$ when the risk threshold increases from $9.14 \times 10^{-2}$ to $10.34 \times 10^{-2}$. The reason for such phenomenon may be that: when the risk threshold changes, the optimal routing and scheduling plan may also change the corresponding itinerary to meet each demand and the speed of the train running on each service legs in the selected itinerary, which may decrease the total risk. 


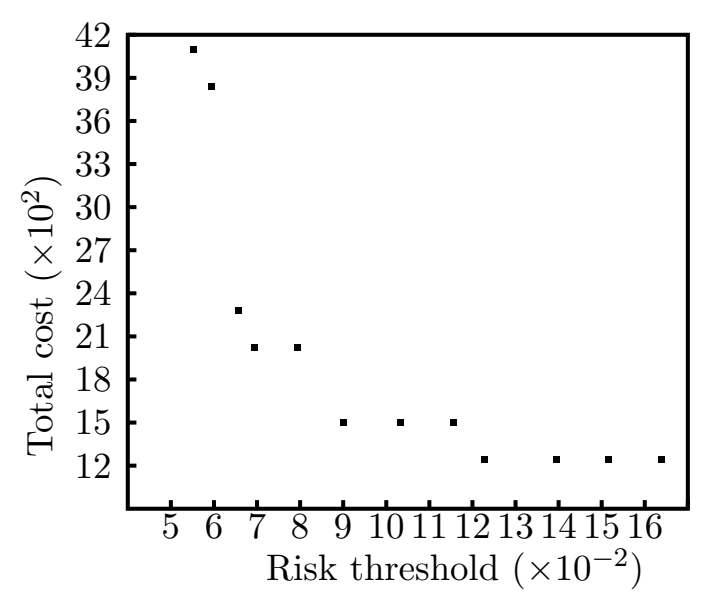

Figure 3: Trade-off between total cost and risk thresh- Figure 4: Relationship between total risk and risk old.

\subsubsection{Impact of train speed}

In an effort to evaluate the impact of train speed on the routing and scheduling plans, we allow a train to assume one of the available speeds on each service leg rather than travel at constant speed on all service legs. It is expected that the routing and scheduling plans are more flexible when speed varies than the case with fixed train speeds. This flexibility may also reduce the total cost. To illustrate, we compare the total cost generated by three different speed schemes:

1. a train can run at alternative speeds within the given speed set;

2. a train can only run at its minimum speed on each service leg; and

3. a train can only run at its maximum speed on each service leg.

We denote their costs as $C_{1}, C_{2}$, and $C_{3}$ respectively. Table 6 shows the costs generated by the above three schemes, in which the cost of the first scheme is comparatively much smaller than the other two speed schemes. As a result, under the pressure of rapidly increasing number of demands, it may be a necessity for the railroad carriers to upgrade their track classes, so that more alternative speeds are available to reduce their total cost.

\subsubsection{Impact of demand due date}

To illustrate the effect of demand due dates on the total cost, we divide the delivery time range $[\underline{L}, \bar{L}]$ of the given origin-destination pair in the above example instance (i.e. 
Table 6: Comparison of the total costs generated by different speed schemes

\begin{tabular}{c|c|cccccc}
\hline \multirow{2}{*}{ speed scheme } & \multirow{2}{*}{ Cost } & \multicolumn{6}{|c}{ Risk threshold $\left(\times 10^{-2}\right)$} \\
\cline { 3 - 8 } & & 5.52 & 5.94 & 6.57 & 6.93 & 9.00 & 12.27 \\
\hline Alternative speeds & $C_{1}$ & 4095 & 3836 & 2282 & 2023 & 1505 & 1246 \\
Minimum speeds & $C_{2}$ & 16786 & 16786 & 16786 & 16786 & 14756 & 14756 \\
Maximum speeds & $C_{3}$ & 19460 & 18914 & 20916 & 21399 & 23863 & 24402 \\
\hline
\end{tabular}

Evansville $\rightarrow$ Lima) into 10 subintervals with the same length $z=(\bar{L}-\underline{L}) / 10$, that is, the $i$ th subinterval is $[\underline{L}+(i-1) z, \underline{L}+i z]$. Thus, varying $i$ from 1 to 10 , we produce ten different instances in which the demand due dates are randomly generated within the $i$ th subinterval, with other things being equal. For each instance, the corresponding cost minimization problem is solved under three different levels of risk thresholds: $5.52 \times 10^{-2}$, $6.93 \times 10^{-2}$, and $12.27 \times 10^{-2}$. Figure 5 graphically shows the corresponding total cost for each instance under different risk thresholds while varying demand due dates.

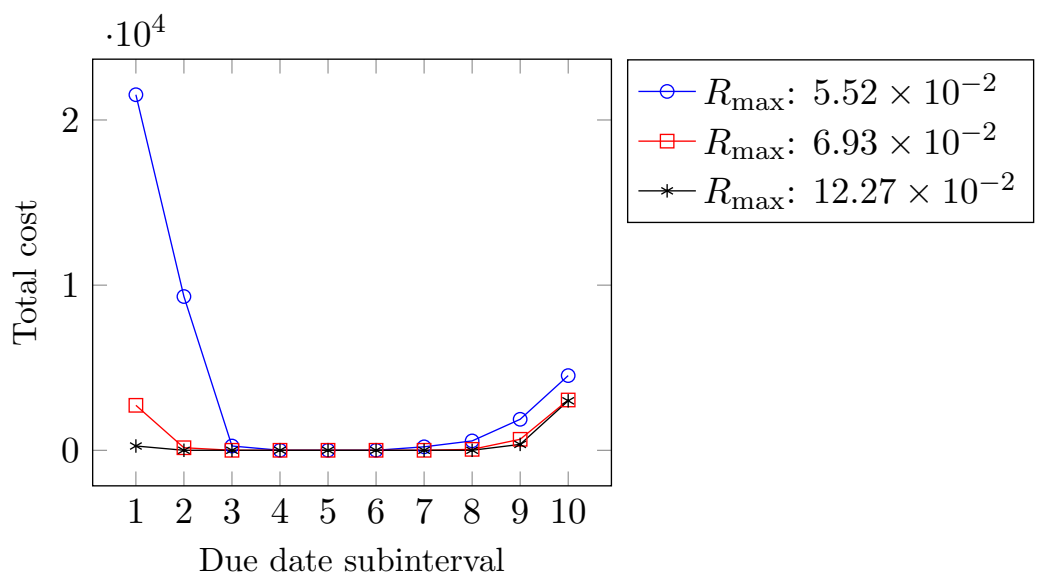

Figure 5: The total cost for the instances under different risk thresholds while varying the demand due date.

From Figure 5, we can see that the total cost decreases substantially when the subinterval of the demand due dates changes from 1 to 3 , keeps at a similar cost level from subintervals 3 to 8 , and increases again from subintervals 8 to 10 . Also note that the more extreme the values of the due date and risk threshold, the more severe impact can been seen on the total cost. The reason behind this situation is quite intuitive. From the aspect of demand due date, in extreme instances, i.e., subintervals 1 (10), the arrival time for most of the demands may be greater (less) than their due dates even if the carrier transports at the maximum 
(minimum) possible speed. Hence a considerable tardiness (earliness) penalty will incur. As the due date subinterval gets less extreme, more scheduling and routing alternatives are available, and therefore, the penalty cost becomes easier to be avoided. This reveals that railroad carriers should suggest moderate delivery times to their customers based on the delivery time range of each demand. The impact from the risk threshold is consistent with the analysis in the previous section.

\subsubsection{Computational time}

We also observe that the CPU running time of solving the mixed integer programming formulation increases rapidly when the number of demands increases. For example, we generate 8 instances randomly with different number of demands, and Figure 6 shows the corresponding CPU running time for solving these instances. When the number of demands exceeds 15 , the randomly generated instance cannot be solved within 2 hours. This observation gives us a strong motivation to design heuristic algorithms to find a feasible solution in reasonable time since the number of demands in real rail transportations is generally large.

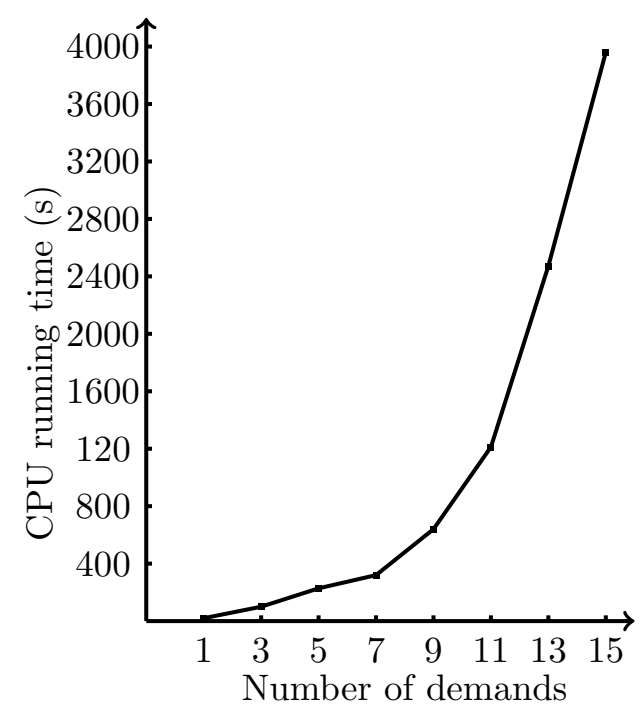

Figure 6: Relationship between running time and the number of demands for solving the MIP. 


\subsection{Experiment 2: Performance of the TSLB heuristic algorithm}

\subsubsection{Performance measurements}

From Experiment 1, we know that when the number of demands exceeds 15, it is time consuming or even unable to solve the proposed mixed integer program within a reasonable time. In this experiment, we focus on the performance of the heuristic algorithm TSLB. Consider the following set of the number of demands:

$$
m \in\{10,20,50,100,150,200,300,400,600,800\} .
$$

For each number of demands $m$, we randomly generate 50 instances, i.e. for a total of $10 \times 50=500$ instances, on the given network (see Figure 2). For each instance, the risk threshold is generated randomly within its risk threshold range, and different demands may have different origin-destination pairs.

For the sake of simplicity, we denote $\operatorname{Cost}(\mathrm{LB})_{i}$ and $\operatorname{Cost}(\mathrm{TSLB})_{i}$ as the corresponding costs by the lower bounding scheme and Algorithm TSLB for each instance $i$, respectively. Note that when $\operatorname{Cost}(\mathrm{LB})_{i}=\operatorname{Cost}(\mathrm{TSLB})_{i}$, Algorithm TSLB must obtain the optimal solution for instance $i$. Let $N_{\mathrm{opt}}^{\mathrm{TSLB}}$ be the numbers of instances that Algorithm TSLB obtain optimal solutions.

For those instances where $\operatorname{Cost}(\mathrm{LB}) \neq \operatorname{Cost}(\mathrm{TSLB})$, we measure the performance of Algorithm TSLB in the following two ways:

1. $\operatorname{Cost}(\mathrm{LB})=0$. In this case, we calculate the maximum time units of earliness $\left(E_{\max }\right)$, lateness $T_{\max }$ and the total idle time $H_{\text {total }}$ by Algorithm TSLB among those instances with $\operatorname{Cost}(\mathrm{LB})=0$ for each number of demand, to measure the deviation from the lower bounding scheme.

2. $\operatorname{Cost}(\mathrm{LB})>0$. In this case, we denote $\mathcal{N}_{\mathrm{LB}}^{+}$and $N_{\mathrm{LB}}^{+}$as the set of and the number of instances that have positive values of $\operatorname{Cost}(\mathrm{LB})$ respectively, and calculate the average ratio between Algorithm TSLB and the lower bounding scheme among those instances, that is,

$$
\text { average ratio }=\left(\sum_{i \in \mathcal{N}_{\mathrm{LB}}^{+}} \frac{\operatorname{Cost}(\mathrm{TSLB})_{i}}{\operatorname{Cost}(\mathrm{LB})_{i}}\right) / N_{\mathrm{LB}}^{+} .
$$

Table 7 shows the performance of Algorithm TSLB. From this table, we can see that when the number of demands is small, the algorithm outputs a reasonable numbers of 
optimal solutions (42 out of 50 for $m=50$ ). When Algorithm TSLB does not output an optimal solution, the ratio between its cost and the lower bounding cost is fairly small (1.088 for $m=100$ ). When the number of demands increases, the number of optimal solutions by Algorithm TSLB decreases. In addition, the average ratio between Cost(TSLB) and Cost(LB) increases slowly (the ratio increases from 1.000 for $m=10$ to 1.844 for $m=800$ ). However, the maximum ratio among all the 50 instances increases much faster than the average ratio when the number of demands become large (3.382 for $m=800)$, which means that Algorithm TSLB may incur undesirable routing and scheduling plans for some extreme instances (e.g. multiple demands share common service legs).

Table 7: Performance of Algorithm TSLB

\begin{tabular}{cc|ccc|cccc}
\hline$m$ & $N_{\mathrm{opt}}^{\mathrm{TSLB}}$ & $E_{\max }^{\mathrm{TSLB}}$ & $T_{\max }^{\mathrm{TSLB}}$ & $H_{\text {total }}^{\mathrm{TSLB}}$ & $N_{\mathrm{LB}}^{+}$ & Avg ratio & Min ratio & Max ratio \\
\hline 10 & 49 & 0 & 1 & 0 & 33 & 1.000 & 1.000 & 1.000 \\
20 & 48 & 0 & 0 & 0 & 48 & 1.005 & 1.000 & 1.121 \\
50 & 42 & - & - & - & 50 & 1.042 & 1.000 & 2.004 \\
100 & 28 & - & - & - & 50 & 1.088 & 1.000 & 1.563 \\
150 & 17 & - & - & - & 50 & 1.107 & 1.000 & 2.560 \\
200 & 11 & - & - & - & 50 & 1.143 & 1.000 & 1.702 \\
300 & 0 & - & - & - & 50 & 1.312 & 1.036 & 2.105 \\
400 & 0 & - & - & - & 50 & 1.444 & 1.067 & 2.537 \\
600 & 0 & - & - & - & 50 & 1.593 & 1.046 & 2.869 \\
800 & 0 & - & - & - & 50 & 1.844 & 1.192 & 3.382 \\
\hline
\end{tabular}

$-:$ no instance with $\operatorname{Cost}(\mathrm{LB})=0$.

Note that we used the lower bound obtained by Algorithm 5.2 to approximate the optimal value in the calculation of the performance ratio, thus the actual performance of Algorithm TSLB could be better than the results in Table 7 . To reduce the performance ratio of our proposed heuristic algorithm, especially for those extreme instances, two possible directions may be further investigated: 1) does our proposed formulation have strong LP relaxations or tighter lower bounds? 2) can Algorithm TSLB be improved by using different selection schemes of the demands that need to be delayed at yards?

\subsubsection{Impact of risk threshold}

Note that the performance of Algorithm TSLB closely depends on the value of the given risk threshold, in this section, we investigate the performance of Algorithm TSLB on the same 50 instances that with $m=200$ as in Section 7.4.1, except that for each instance $i$, we divide its risk threshold range $\left[\underline{R}_{i}, \bar{R}_{i}\right]$ into 10 subintervals with the same length 
$z_{i}^{\prime}=\left(\bar{R}_{i}-\underline{R}_{i}\right) / 10$, that is, the $j$ th subinterval is $\left[\underline{R}_{i}+(j-1) z_{i}^{\prime}, \underline{R}_{i}+j z_{i}^{\prime}\right]$. By varying $j$ from 1 to 10 , for each instance $i$, we produce ten different instances in which the risk threshold are randomly generated within the $j$ th subinterval. Figure 7 shows the corresponding performance measurements while varying the risk threshold subintervals.

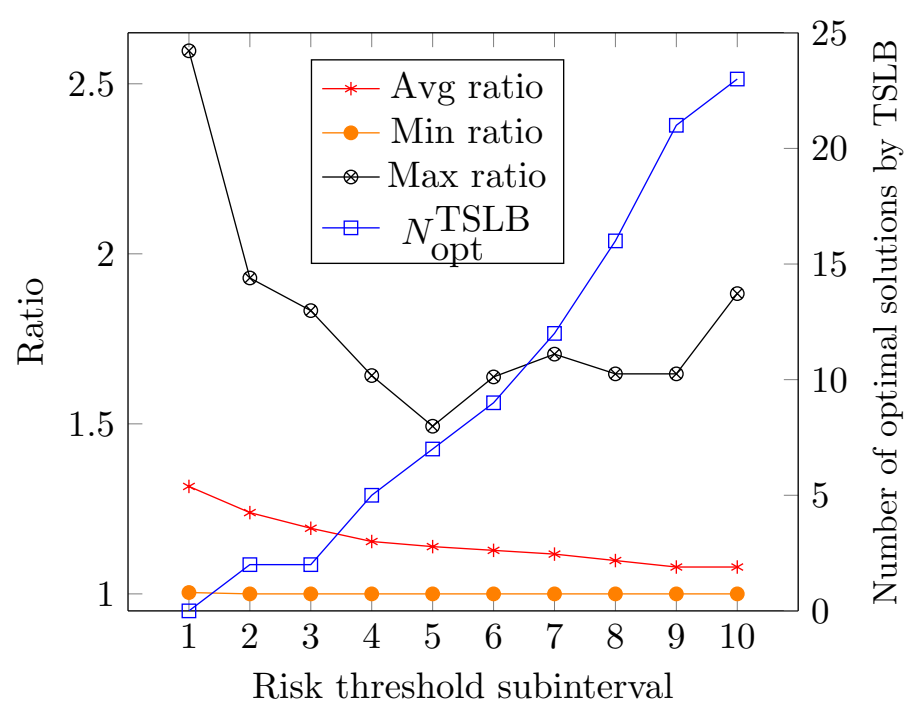

Figure 7: The performance measurements for the instances with $m=200$ while varying the risk threshold.

From Figure 7, we can see that when the risk threshold subinterval increases, the average ratio decreases from 1.316 to 1.079 (the line with asterisk marks), while the number of optimal solutions obtained by Algorithm TSLB increases from 0 to 23 (the line with square marks). This reveals that the higher the risk threshold, the better the performance of Algorithm TSLB.

\subsubsection{Computational time}

As we know that it is time consuming to obtain an exact solution by solving the proposed mixed integer programming. It is shown that the CPU running time for implementing Algorithm TSLB is fairly small. Figure 8 shows the average CPU running time of Algorithm TSLB for each instance in terms of different numbers of demands. Note that even when the number of demands is 800 , the average running time for solving each instance is only about 3 minutes. This fact suggests that Algorithm TSLB is a simple and intuitive, yet powerful heuristic algorithm, which provides good results in practice, both on its outputs and its acceptable running time. 


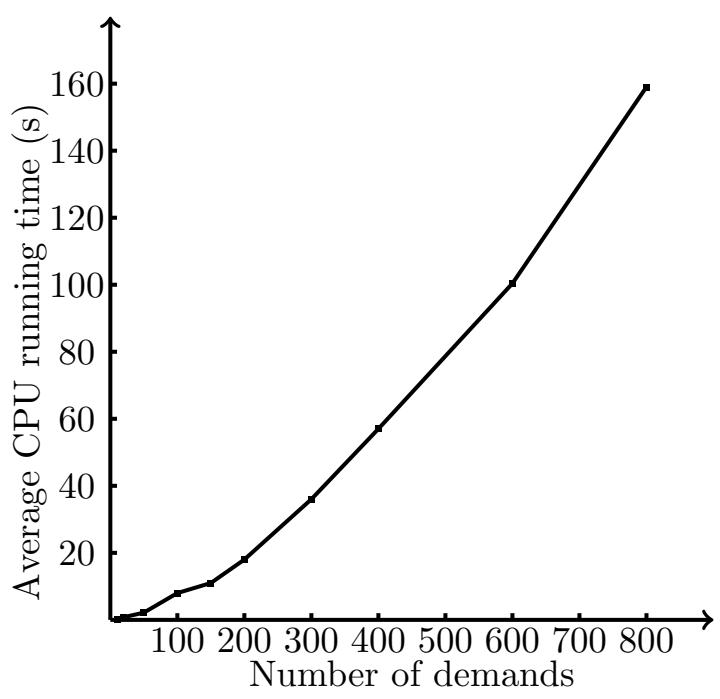

Figure 8: Relationship between running time and the number of demands for Algorithm TSLB.

\section{Conclusions}

In this work, we consider the problem of minimizing the weighted sum of earliness and tardiness for each demand and the holding cost at each yard, under risk threshold constraints. Analysis of FRA accident data underlined the importance of train speed in derailment. We propose a mixed integer programming formulation and prove that this problem is strongly NP-hard. A lower bounding scheme and a heuristic algorithm (Algorithm TSLB) is designed, and the corresponding computational performance are investigated with instances arising from randomly generated demands and real-life rail networks. Through computational experiments we could conclude that cost savings can be accomplished either by upgrading the track classes or by imposing higher risk thresholds on service legs. It is also shown that Algorithm TSLB performs very well for medium and large instances, while solving the mixed integer programming formulation can be very time consuming.

There are many possible directions for future research stemming from this work. One natural direction would be to investigate other hazmat transportation problems with demand due date considerations, e.g., the truck routing problem in the highway system. It is important to note that the proposed framework would require adaptations to capture the characteristics of highway transportation. For example, the model would need to consider the fact that trucks can travel at varying speed on a given stretch of road. In addition, the 
model should be adapted to do away with the processing time at intermediate locations. Finally, one would need to undertake primary research to establish a relationship between truck accidents and pertinent factors.

Another direction would be to investigate tighter lower bounding schemes and design new heuristic algorithms to improve the performance ratio. It would also be interesting to consider additional types of train costs, such as the cost related to the number of trains used, the setup cost of using specific itineraries, and the yard operations cost, to name a few, or to investigate how to set the appropriate risk threshold levels from the perspective of regulatory authorities.

\section{Acknowledgements}

This research has been supported in part by a grant from the National Sciences and Engineering Research Council of Canada (Grant \# 312936), a Discovery Grant from the Natural Sciences and Engineering Research Council of Canada (Grant\# RGPIN-201504013), and a grant from the National Science Foundation of China (Grant \# 91646118). The third author is a member of the Interuniversity Research Center on Enterprise, Network Logistics and Transportation (CIRRELT) and acknowledge the research infrastructure provided by the Center.

\section{Appendix: NP-hardness of the RTHD problem}

Proof. We reduce any instance of the Minimum Weighted Sum Bin Packing (MWSBP) problem, which is strongly NP-hard (Epstein and Levin, 2008), to an instance of the RTHD problem. The MWSBP problem is described as follows: Given a set of items $\mathcal{M}=\{1,2, \ldots, m\}$, where item $i$ has a size $h_{i}$ and a weight $\psi_{i}$, does there exist a partition of $\mathcal{M}$ into $\mathcal{M}_{1}, \mathcal{M}_{2}, \ldots, \mathcal{M}_{t}$, such that for all $j=1,2, \ldots, t, \sum_{i \in \mathcal{M}_{j}} h_{i} \leq R$, and the objective $\sum_{j=1}^{t} j\left(\sum_{i \in \mathcal{M}_{j}} \psi_{i}\right)$ is minimized?

Given any instance $\mathcal{I}_{1}$ of the MWSBP problem, we construct an instance $\mathcal{I}_{2}$ of the RTHD problem as follows. The rail network $\mathcal{G}=(\mathcal{C}, \mathcal{L})$ is represented in Figure 9, in which $\mathcal{C}=\left\{A, B, Y_{1}, \ldots, Y_{m}\right\}$ and $\mathcal{L}=\left\{\ell^{\prime}, \ell_{1}, \ldots, \ell_{m}\right\}$. There is a set of demands $\mathcal{M}=$ $\{1,2, \ldots, m\}$, where demand $i$ departs at yard $A$ and arrives at yard $Y_{i}$. From the network structure, it is easy to see that there exists only one available itinerary to meet each demand. All of the demands have a common due date $d_{i}=0$ for any $i \in \mathcal{M}$. The number of hazmat and regular railcars required for each demand $i$ are $h_{i}$ and 0 respectively. The tardiness and 
earliness penalty costs per unit time and per railcar for $i \in \mathcal{M}$ are $\psi_{i} / h_{i}$ and 0 respectively. The processing time and the holding penalty cost per unit time and per railcar at each yard are both 0 . The train can only run at a uniform speed. The traveling time on service leg $\ell^{\prime}$ is $p_{\ell^{\prime}}=0$ and the traveling time on service leg $\ell_{i}$ is $p_{\ell_{i}}=1(i=1, \ldots, m)$. The risk incurred for a train running on service leg $\ell^{\prime}$ and $\ell_{i}$ are $r_{\ell^{\prime}}=r_{\ell_{i}}=1$, and the risk threshold on any service leg at any time instant is $R_{\max }=R$.

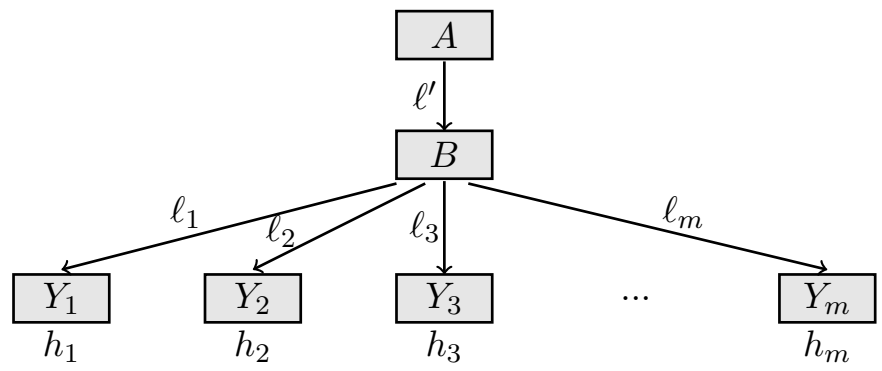

Figure 9: The rail network used in the proof of NP-hardness.

Note that the earliness and holding costs for instance $\mathcal{I}_{2}$ of the RTHD problem is 0 , so we only need to consider minimizing the total tardiness cost. Since all the demands have a common due date 0 , we should fulfill the demands as early as possible to minimize the total tardiness cost. Note that $p_{\ell^{\prime}}=0$ and $p_{\ell_{i}}=1$, so we know that the finish time for any demand is at least 1 . However, under the restriction on the given risk threshold, we may not be able to finish all the demands simultaneously at time 1 . Let $\mathcal{M}_{j}$ be the set of demands that were finished at time instant $j$. In order to minimize the cost, we have to partition all the demands according to their finish time into $\mathcal{M}_{1}, \ldots, \mathcal{M}_{t}$ such that for all time instances $j=1,2, \ldots, t$, the risk incurred on each service leg at any time instant $j$ is no greater than the given risk threshold, that is, the total risk incurred on service leg $\ell^{\prime}$ at time instant $j$ should satisfy $r_{\ell^{\prime}}\left(\sum_{i \in \mathcal{M}_{j}} h_{i}\right)=\sum_{i \in \mathcal{M}_{j}} h_{i} \leq R$. The tardiness of each demand $i \in \mathcal{M}_{j}$ is $j-0=j$, so the total tardiness cost for set $\mathcal{M}_{j}$ is $j\left(\sum_{i \in \mathcal{M}_{j}} h_{i} \times\left(\psi_{i} / h_{i}\right)\right)=j\left(\sum_{i \in \mathcal{M}_{j}} \psi_{i}\right)$. As a result, the objective is to minimize $\sum_{j=1}^{t} j\left(\sum_{i \in \mathcal{M}_{j}} \psi_{i}\right)$. Therefore, we construct a reduction from the MWSBP problem to the RTHD probelm, and therefore the RTHD problem is strongly NP-hard. 


\section{References}

\section{References}

AAR, December 2013. Moving crude by rail. Tech. rep., Association of American Railroads.

Abkowitz, M., Lepofsky, M., Cheng, P., 1992. Selecting criteria for designating hazardous materials highway routes. Transportation Research Record 1333, 30-35.

Agustina, D., Lee, C., Piplani, R., 2014. Vehicle scheduling and routing at a cross docking center for food supply chains. International Journal of Production Economics 152 (2), $29-41$.

Akgün, V., Erkut, E., Batta, R., 2000. On finding dissimilar paths. European Journal of Operations Research 121 (2), 232-246.

Alp, E., 1995. Risk-based transportation planning practice: overall methodology and a case example. INFOR 33 (1), 4-19.

Anderson, R., Barkan, C., 2004. Railroad accident rates for use in transportation risk analysis. Transportation Research Record 1863, 88-98.

Arcview, 1996. Geographical information system. http://www.esri.com.

Arya, S., 1999. Air Pollution Meteorology and Dispersion. Oxford University Press, New York.

Assadipour, G., Ke, G., Verma, M., 2015. Planning and managing intermodal transportation of hazardous materials with capacity selection and congestion. Transportation Research Part E: Transportation and Logistics Review 76, 45-57.

Bagheri, M., Verma, M., Verter, V., 2014. Transport mode selection for toxic gases: rail or road? Risk Analysis 34 (1), 168-186.

Barkan, C., 2008. Improving the design of higher-capacity railway tank cars for hazardous materials transport: optimizing the trade-off between weight and safety. Journal of Hazardous Materials 160 (1), 122-134.

Barkan, C., Dick, C., Anderson, R., 2003. Analysis of railroad derailment factors affecting hazardous materials transportation risk. Transportation Research Record 1825, 64-74. 
Barkan, C., Treichel, T., Widell, G., 2000. Reducing hazardous materials releases from railroad tank car safety vents. Transportation Research Record 1707, 27-34.

Barkan, C., Ukkusuri, S., 2007. Optimizing railroad tank cars for safety: the tradeoff between damage resistance and probability of accident involvement. Computers \& Operations Research 34, 1266-1286.

Batta, R., Chiu, S., 1988a. Optimal obnoxious paths on a network: transportation of hazardous materials. Operations Research 36 (1), 84-92.

Batta, R., Chiu, S., 1988b. Optimal obnoxious paths on a network: transportation of hazardous materials. Operations Research 36 (1), 84-92.

Bell, M., 2006. Mixed route strategies for the risk-averse shipment of hazardous materials. Networks and Spatial Economic 6 (3-4), 253-265.

Bianco, L., Caramia, M., Giordani, S., 2009. A bilevel flow model for hazmat transportation network design. Transportation Research Part C: Emerging Technologies 17 (2), 175-196.

Bianco, L., Caramia, M., Giordani, S., Piccialli, V., 2016. A game-theoretic approach for regulating hazmat transportation. Transportation Science 50 (2), 424-438.

Bülbül, K., Philip, K., Candace, Y., 2004. Flow shop scheduling with earliness, tardiness and intermediate inventory holding costs. Naval Research Logistics 51 (3), 407-445.

CAPP, June 2014. Crude Oil: Forecast, Market and Transportation. http://www.capp.ca.

Carotenuto, P., Giordani, S., Ricciardelli, S., 2007a. Finding minimum and equitable risk routes for hazmat shipments. Computers and Operations Research 34 (5), 1304-1327.

Carotenuto, P., Giordani, S., Ricciardelli, S., Rismondo, S., 2007b. A tabu search approach for scheduling hazmat shipments. Computers and Operations Research 34 (5), 13281350.

Christiansen, M., Fagerholt, K., 2002. Robust ship scheduling with multiple time windows. Naval Research Logistics 49 (6), 611-625.

Cordeau, J.-F., Toth, P., Vigo, D., 1998. A survey of optimization models for train routing and scheduling. Transportation Science 32 (4), 380-404.

Covello, V., Merkhofer, M., 1993. Risk Assessment Methods: Approaches for Assessing Health and Environmental Risks. Plenum Publishing Corporation, New York. 
DOT, 2007. Hazardous materials incident data: office of hazardous materials safety. US Department of Transportation, Washington, DC.

Epstein, L., Levin, A., 2008. Minimum weighted sum bin packing. In: Kaklamanis, C., Skutella, M. (Eds.), Approximation and Online Algorithms. Vol. 4927 of Lecture Notes in Computer Science. Springer, pp. 218-231.

Erkut, E., Tijandra, S., Verter, V., 2007. Hazardous materials transportation. In: Barnhart, C., Laporte, G. (Eds.), Handbooks in Operations Research and Management Science. Vol. 14. Elsevier, pp. 539-621.

Erkut, E., Verter, V., 1998. Modeling of transport risk for hazardous materials. Operations Research 46 (5), 625-642.

FRA, 2013. Accident Data on Demand: Federal Railroad Administration Office of Safety Analysis. http://safetydata.fra.dot.gov/officeofsafety.

Garey, M., Johnson, D., 1979. Computers and Intractability: A Guide to the Theory of NP-Completeness. W.H. Freeman, New York.

Glickman, T., 1983. Rerouting railroad shipments of hazardous materials to avoid populated areas. Accident Analysis \& Prevention 15 (5), 329-335.

Glickman, T., Erkut, E., Zschocke, M., 2007. The cost and risk impacts of rerouting railroad shipments of hazardous materials. Accident Analysis \& Prevention 39 (5), 1015-1025.

Glickman, T., Rosenfield, D., 1984. Risks of catastrophic derailments involving the release of hazardous materials. Management Science 30 (4), 503-511.

Gopalan, R., Batta, R., Karwan, M., 1990a. The equity constrained shortest path problem. Computers \& Operations Research 17 (3), 297-307.

Gopalan, R., Kolluri, K., Batta, R., Karwan, M., 1990b. Modeling equity of risk in the transportation of hazardous materials. Operations Research 38 (6), 961-975.

Johnson, D. B., Mizoguchi, T., 1978. Selecting the $K$ th element in $X+Y$ and $X_{1}+X_{2}+$ $\cdots+X_{m}$. SIAM Journal on Computing 7 (2), 147-153.

Kallehauge, B., 2008. Formulations and exact algorithms for the vehicle routing problem with time windows. Computers \& Operations Research 35 (7), 2307-2330. 
Kang, Y., Batta, R., Kwon, C., 2014. Generalized route planning model for hazardous material transportation with var and equity considerations. Computers and Operations Research 43, 237-247.

Kwon, O., Martland, C., Sussman, J., 1998. Routing and scheduling temporal and heterogeneous freight car traffic on rail networks. Transportation Research Part E: Transportation and Logistics Review 34 (2), 101-115.

Linder-Dutton, L., Batta, R., Karwan, M., 1991. Equitable sequencing of a given set of hazardous materials shipments. Transportation Science 25 (2), 124-137.

Liu, X., Saat, M., Barkan, C., 2012. Analysis of causes of major train derailment and their effect on accident rates. Transportation Research Record 2289, 154-163.

Miller-Hooks, E., Mahmassani, H., 1998. Optimal routing of hazardous materials in stochastic time-varying transportation networks. Transportation Research Record 1645, 143-151.

Nozick, L., List, G., Turnquist, M., 1997. Integrated routing and scheduling in hazardous materials transportation. Transportation Science 31 (3), 200-215.

Oggero, A., Darbra, R., Munoz, M., Planas, E., Casal, J., 2006. A survey of accidents occurring during the transport of hazardous substances by road and rail. Journal of Hazardous Materials 133 (A), 1-7.

Patel, M., Horowitz, A., 1994. Optimal routing of hazardous materials considering risk of spill. Transportation Research Part A: Policy and Practice 28 (2), 119-132.

Provencher, M., 2008. The movement and handling of dangerous goods in canada for the year 2004. Dangerous Goods Directorate: Transport Canada, Ottawa.

Raj, P., Pritchard, E., 2000. Hazardous materials transportation on US railroads. Transportation Research Record 1707, 22-26.

ReVelle, C., Cohon, J., Shobrys, D., 1991. Simultaneous siting and routing in the disposal of hazardous wastes. Transportation Science 25 (2), 138-145.

Romero, N., Nozick, L., Xu, N., 2016. Hazmat facility location and routing analysis with explicit consideration of equity using the gini coefficient. Transportation Research Part E: Transportation and Logistics Review 89, 165-181. 
Saat, M., Barkan, C., 2005. Release risk and optimization of railroad tank car safety design. Transportation Research Record 1916, 78-87.

Saat, M., Barkan, C., June 2006. The effect of rerouting and tank car safety design on the risk of rail transport of hazardous materials. In: Proceedings of the 7th World Congress on Railway Research. Montréal, Canada.

Saccomanno, F., Chan, A., 1985. Economic evaluation of routing strategies for hazardous road shipments. Transportation Research Record 1020, 12-18.

Sivakumar, R., Batta, R., 1994. The variance-constrained shortest path problem. Transportation Science 28 (4), 390-416.

Verma, M., 2009. A cost and expected consequence approach to planning and managing railroad transportation of hazardous materials. Transportation Research Part D: Transport and Environment 14 (5), 300-308.

Verma, M., 2011. Railroad transportation of dangerous goods: a conditional exposure approach to minimize transport risk. Transportation Research Part C: Emerging Technologies 19 (5), 790-802.

Verma, M., Verter, V., 2007. Railroad transportation of dangerous goods: population exposure to airborne toxins. Computers \& Operations Research 34 (5), 1287-1303.

Verma, M., Verter, V., 2010. A lead-time approach to planning rail-truck intermodal transportation of dangerous goods. European Journal of Operations Research 202, 696706.

Verma, M., Verter, V., 2013. Railroad transportation of hazardous materials: models for risk assessment and management. In: Batta, R., Kwon, C. (Eds.), Handbook of OR/MS Models in Hazardous Materials Transportation. Vol. 193. Springer, pp. 9-47.

Verma, M., Verter, V., Gendreau, M., 2011. A tactical planning model for railroad transportation of dangerous goods. Transportation Science 45 (2), 163-174.

Verma, M., Verter, V., Zufferey, N., 2012. A bi-objective model for planning and managing rail-truck intermodal transportation of hazardous materials. Transportation Research Part E: Transportation and Logistics Review 48 (1), 132-149. 
Verter, V., Kara, B., 2008. A path-based approach for hazmat transport network design. Management Science 54 (1), 29-40.

Wang, W., Fung, R., Chai, Y., 2004. Approach of just-in-time distribution requirements planning for supply chain management. International Journal of Production Economics 91 (2), 101-107. 